\title{
Renormalization of Lattice Feynman Integrals with Massless Propagators
}

\author{
Thomas Reisz \\ Deutsches Elektronen-Synchrotron DESY, D-2000 Hamburg, Federal Republic of Germany \\ Max Planck Institut für Physik und Astrophysik, D-8000 München, \\ Federal Republic of Germany *
}

\begin{abstract}
A renormalization procedure is proposed which applies to lattice Feynman integrals containing zero-mass propagators and is analogous to the BPHZL renormalization procedure for continuum Feynman integrals. The renormalized diagrams are infrared convergent for non-exceptional external momenta, if the vertices of the theory satisfy a general infrared constraint. Under the same conditions as in the massive case [4], the continuum limit of the renormalized theory exists and is independent of the details of the lattice action.
\end{abstract}

\section{Introduction}

Feynman integrals with a lattice cutoff have a very specific structure. They are absolutely convergent for finite lattice spacing, if all propagators are massive. The continuum limit behavior of such diagrams is described by a lattice power counting theorem [3], which uses a new kind of an ultraviolet (UV) divergence degree (the well known power counting theorems of Weinberg [1] and of Hahn and Zimmermann [2] do not apply to diagrams with a lattice cutoff). On the basis of such a power counting theorem a renormalization program for lattice field theories has been given [4], which is analogous to the BPHZ finite part prescription for continuum Feynman integrals [5].

These methods work for massive field theories. In the presence of massless fields, additional arguments are needed to avoid infrared (IR) divergencies. It has been shown [6] that the UV-power counting conditions only have to be supplemented by IR-power counting conditions, and IR-singularities are tractable by the same methods as in the continuum $[7,8]$. In this article, we use this power counting to give a renormalization procedure for lattice Feynman integrals with massless propagators.

\footnotetext{
$\star$ Present address
} 
In outline, the idea of the construction is as follows. As in the massive case, the continuum limit is controlled by UV-divergence degrees. As a convergence condition, they should always be less than zero. This can be achieved by appropriate subtractions. However, in the presence of massless propagators, subtractions at zero momenta are no longer IR-convergent. The IR-divergencies can be avoided by choosing the subtraction points at non-exceptional momenta, and by additional finite renormalizations, which are chosen in such a way that in the sum of all diagrams to a given order all IR-singularities drop out. For example, in a gauge theory the renormalized coupling may be defined as the value of an appropriate vertex function at non-exceptional momenta, whereas the self-energy of the gauge field has only a wave function renormalization and vanishes for zero external momentum. However, when we want to renormalize diagrams separately by the forest formula, we run into the problem of IR-singularities also if we choose normalization points at non-exceptional momenta (cf. Sect. 2.1). For instance, to make a two-point diagram UV-convergent, in general two differentiations are necessary. This produces an IR-singularity by differentiating a propagator twice.

To prove the convergence of a renormalization procedure we shall use the power counting theorem of [6]. This necessitates all subtractions and differentiations being collected in the integrand, leading to a forest-formula like expression. As indicated above, this induces IR-divergencies also for subtractions at nonexceptional momenta. A possibility to overcome this problem is to introduce auxiliary masses in the counterterms. This means we employ the (lattice-modified) BPHZL renormalization procedure of Lowenstein and Zimmermann $[9,10]$. Propagators of a bare mass $\mu$ (which may be zero) get a mass-dependence of the form

$$
\mu^{2}+(s-1)^{2} M^{2}, \quad \mu^{2}+M^{2}>0,
$$

$s$ is called the mass parameter. Counterterms are now constructed for $s=0$, and after all subtractions are done we set $s=1$, so that we get a renormalized theory of the original model. Two important points must be taken into account.

1. Due to the auxiliary mass dependence of the counterterms, to get all UVdivergence degrees smaller than zero, differentiations not only with respect to the external momenta but also with respect to the mass parameter $s$ are necessary. This means that subtractions are combinations of lattice subtraction operators and Taylor polynomials in $s$. They will be called generalized subtraction operators.

2. Additional finite renormalizations are necessary to avoid IR-singularities by renormalized subdiagrams. For instance, inserting a self-energy subgraph into a massless line usually produces a non-integrable singularity. This difficulty is solved by imposing a normalization condition so that such a diagram vanishes for zero external momenta and $s=1$.

Both conditions are satisfied if instead of the subtraction operator $\hat{t}_{q}^{\delta}[4]$ we employ

$$
\hat{t}_{q(s-1)}^{\varrho-1}+\left(1-\hat{t}_{q(s-1)}^{\varrho-1}\right) \hat{t}_{q s}^{\delta}
$$

$\varrho$ being the IR-divergence degree of the diagram, and the $\hat{t}$ are generalized subtraction operators. Using these subtractions, the renormalized theory is IR- 
finite for all $s$, including the case $s=1$, and the continuum limit exists if

1. the external momenta are non-exceptional, and

2. $r(V) \geqq 4$ for all internal vertices $V$,

where $r(V)$ is the (lattice-) IR-degree of the vertex $V$ (defined below). An internal vertex is one with no external line. The latter constraint restricts the class of renormalizable, IR-finite theories. For instance, a massless $\Phi^{3}$-theory is IRdivergent in four-dimensions $\left(r\left(\Phi^{3}\right)=3\right)$. Note that we have made no statement about the IR-behavior of the bare theory. The renormalized, massless $\Phi^{4}$-theory is IR-convergent, but the bare theory is not.

In Sect. 2.1 we give a 1-loop example which should show the efficiency of the auxiliary mass method. The reader who is familiar with the method may skip this subsection. In the remainder of Sect. 2, general notations concerning Feynman diagrams with an arbitrary number of loops are given. They are essentially the same as in the massive case [4], and we only sketch the most important ones. Furthermore, generalized notions of infrared and ultraviolet lattice divergence degrees are introduced. Due to the introduction of the mass parameter $s$, this generalization of the lattice divergence degrees defined in [3] and [6] is necessary. Finally, the definition of a generalized subtraction operator (GSO) is given. The main theorem which describes the renormalization of lattice Feynman integrals and lattice Green functions is given in Sect. 3. In Sect. 4, important properties of GSO's are given. In the remainder of this article, the theorem is proved, using the properties of GSO's and the power counting theorem of [6], by showing that all UV- and IR-power counting conditions of this theorem are satisfied.

\section{The Auxiliary Mass Method and Generalized Subtraction Operators}

\subsection{A One Loop Example}

Before we are going to define the renormalization prescription to every order, we shall consider the auxiliary mass method for the one loop case. To be specific, consider the scalar $\Phi^{4}$-theory. The propagator is given by

$$
\widehat{\Delta}(k ; s, a)=\frac{1}{\hat{k}^{2}+(s-1)^{2} M^{2}},
$$

where

$$
\widehat{k}_{i}=\frac{2}{a} \sin \frac{k_{i} a}{2}, \quad i=1, \ldots, 4, \quad \hat{k}^{2}=\sum_{i=1}^{4}\left(\widehat{k}_{i}\right)^{2},
$$

and $M \neq 0$ is an auxiliary mass, $a$ denotes the lattice spacing. For $s=1$, the propagator is massless. A one loop contribution to the four-point function is of the form (Fig. 1)

$$
\hat{I}(q ; s, a)=\int_{-\pi / a}^{\pi / a} \frac{d^{4} k}{(2 \pi)^{4}} \hat{\Delta}(k ; s, a) \hat{\Delta}(k+q ; s, a) .
$$

To renormalize the diagram, following the ideas of the (lattice) BPHZ procedure, one should subtract from the integrand its value at vanishing external momentum 


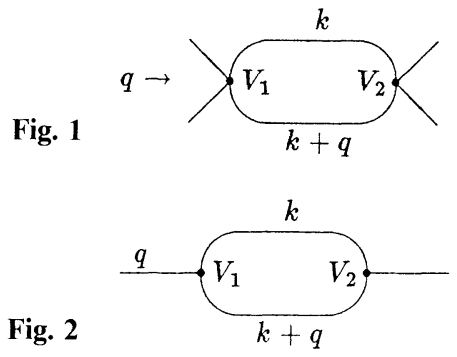

q. However, for $s=1$, this produces a non-integrable IR-singularity, i.e. for the massless theory this method does not work. The idea of Lowenstein and Zimmermann $[9,10]$ consists in subtracting at $q=0$ and simultaneously at $s=0$, i.e. giving the counterterm a mass. For non-vanishing $q$, the renormalized Feynman integral

$$
\hat{R}(q ; s, a)=\int_{-\pi / a}^{\pi / a} \frac{d^{4} k}{(2 \pi)^{4}}\left[\hat{\Delta}(k ; s, a) \hat{\Delta}(k+q ; s, a)-(\hat{\Delta}(k ; 0, a))^{2}\right]
$$

is IR-convergent also for $s=1$, and the continuum limit exists. Summing all contributions of the form (2.4), we get the renormalized four point function at one loop order. It depends on the auxiliary mass $M$. However, this dependence is only exhibited by a momentum independent and finite (i.e. in the continuum limit convergent) term, and hence may be compensated by a finite counterterm to the lattice action of the form

$$
a^{4} \sum_{n \in \mathbb{Z}^{4}} c(M) \Phi^{4}(n a),
$$

which satisfies the IR-constraint alluded to in the introduction. In this way, normalization conditions at non-exceptional momenta may be implemented.

If a diagram having a UV-divergence degree greater than zero is to be renormalized, we clearly have to differentiate not only with respect to the external momenta, but also to the mass parameter s. Otherwise, the UV-divergencies would not be cancelled because of the different mass dependence of the bare and the counterterm integrand. This situation happens e.g. for two-point functions.

One may try to apply subtractions at non-exceptional external momenta instead of using the auxiliary mass method. However, this does not work if subtractions are directly applied to the integrand. To see this, consider the Feynman integral (Fig. 2)

$$
\hat{J}(q ; s, a)=\int_{-\pi / a}^{\pi / a} \frac{d^{4} k}{(2 \pi)^{4}} V_{1}(k, q ; a) V_{2}(k, q ; a) \hat{\Delta}(k ; s, a) \hat{\Delta}(k+q ; s, a),
$$

where the vertex functions $V_{1}, V_{2}$ satisfy

$$
V_{i}(\lambda k, \lambda q ; a)=O(\lambda) \quad \text { as } \quad \lambda \rightarrow 0,
$$

and $\hat{\Delta}$ is given by (2.1). Diagrams of the form (2.5) appear in perturbative lattice gauge theory. The conditions (2.6) insure that the IR-constraints on the vertices are satisfied. 
The UV-divergence degree of $\hat{J}$ is at least two. It can easily be seen that a subtraction operator $\hat{t}_{q}[4]$ does not apply to the integrand of (2.5) without producing a non-integrable singularity, even if we choose a subtraction point $\bar{q} \neq 0$. For, if $\hat{u}(k+q)$ is differentiated twice with respect to $q$ and then $q$ is set equal to $\bar{q}$, we get such a singularity at $k=-\bar{q}$. For this reason, we employ the auxiliary mass method which circumvents this problem. Normalization conditions at nonexceptional momenta may be implemented afterwards by finite counterterm contributions to the lattice action, satisfying the IR-constraint.

Finally, some words are in order concerning the IR-constraints mentioned in the introduction. Consider the Feynman integral (2.5) again, but now set $V_{1}=V_{2}=1$. The vertices then have an IR-degree equal to three (three massless legs). $\hat{J}$ is the one loop contribution to the unrenormalized two-point function in the lattice $\Phi^{3}$-theory. As before, we make the subtraction of order two at $q=0$ and $s=0$. Then $\widehat{J}$ remains IR-finite, and the continuum limit exists. However, inserting (2.5), or its renormalized expression as just described, into a massless line which is integrated over, results in an IR-divergence. Consequently, to get a finite result we should subtract from (2.5) its value at $q=0$. But $\hat{J}(q ; s, a)$ does not exist for $q=0$ and $s=1$, and the same holds for its renormalized form. This means that the massless $\Phi^{3}$-theory is IR-divergent in four dimensions.

If, instead of $V_{1}=V_{2}=1$, the vertices satisfy (2.6), which means that $r\left(V_{1}\right)$ $=r\left(V_{2}\right) \geqq 4$, then $\hat{J}$ exists for $q=0$ and $s=1$. Furthermore, by finite renormalizations, it can always be achieved that diagrams with two massless external lines vanish at zero external momentum. This means that massless bare fields remain massless after renormalization. The same situation occurs for diagrams with three massless external lines. In general, these are the only basic field vertex functions whose overall subtractions imply additional finite renormalizations. They are convergent even for zero external momenta, whereas in general exceptional external momenta must be excluded.

\subsection{Diagrammatic Notations}

We now give some general notations which will be needed later on. In part, they are the same as in [4]. Only the modifications and additions will be pointed out here.

In perturbation theory, a 1PI function, i.e. a one-particle irreducible (1PI) Green function is written as an asymptotic sum of contributions

$$
\left\langle\prod_{i=1}^{N} P_{i}\left(A, n_{i} a\right) \cdot \prod_{j=1}^{M}\left[Q_{j}\right]\right\rangle_{0,1 \mathrm{PI}},
$$

where

$$
\left[Q_{j}\right]=a^{4} \sum_{n \in \mathbb{Z}^{4}} Q_{j}(A, n a)
$$

in general is a contribution of the interaction part of the action. The subscript in (2.7) indicates that (2.7) is the 1PI part of

$$
\frac{1}{Z_{0}} \int \mathscr{D}(A) \prod_{i=1}^{N} P_{i}\left(A, n_{i} a\right) \cdot \prod_{j=1}^{M}\left[Q_{j}\right] \cdot e^{-S_{0}(A)},
$$


where

$$
Z_{0}=\int \mathscr{D}(A) \cdot e^{-S_{0}(A)},
$$

and $\mathscr{D}(A)=\prod_{i, n \in \mathbb{Z}^{4}} d A_{i}(n a) . A$ represents all fields $A_{i}$ and $S_{0}$ is the free part of the action. $P_{i}\left(A, n_{i} a\right)$ and $Q_{i}\left(A, n_{i} a\right)$ are polynomials in the lattice spacing $a$ and the fields $A$ at $n_{i} a$ and neighboring lattice sites, and they are homogeneous in the fields $A$. They represent basic fields or composite operators.

Expression (2.7) is a sum of 1PI Feynman diagrams. We recall that a diagram is called 1 PI if it is connected and does not get disconnected upon cutting any one of its internal lines [4]. Divergencies manifest themselves in 1PI diagrams when the cutoff is removed. Such diagrams must be renormalized. Note that in our notation we distinguish between 1PI functions and vertex functions. The latter are amplitudes which result from a Legendre transformation of the generating functional of connected Green functions. They are not always 1PI, e.g. for theories with spontaneous symmetry breaking. However, every such diagram is mainly a product of 1 PI graphs, and the latter can be renormalized as described below. In particular, tadpole diagrams vanish after renormalization. When we take into account symmetries, the vertex functions must satisfy corresponding Wardidentities. After renormalization of all 1PI functions (to a given order), normalization conditions of vertex functions are to be implemented by additional finite renormalizations of proper functions, satisfying the IR-constraint indicated in the introduction. This must be done very carefully in order not to produce new IRdivergencies.

In the following we consider the $1 \mathrm{PI}$ functions in momentum space

$$
\left\{\prod_{i=1}^{N} \tilde{P}_{i}\left(q_{i}\right) \cdot \prod_{j=1}^{M}\left[Q_{j}\right]\right\}_{0,1 \mathrm{PI}}(2 \pi)^{4} \delta_{a}^{4}\left(\sum_{i=1}^{N} q_{i}\right)
$$

where $\delta_{a}^{4}(Q)=\sum_{m \in \mathbb{Z}^{4}} \delta^{4}\left(Q-\frac{2 \pi}{a} m\right)$ for $Q \in \mathbb{R}^{4}$. Expression (2.9) is a sum of 1PI momentum space Feynman integrals.

In what follows we are using the notations of [4]. Here we only sketch some of them. Let $\Gamma$ be an arbitrary 1PI diagram

$$
\Gamma=\left(\mathscr{L}_{\Gamma}, \mathscr{E}_{\Gamma}, \mathscr{B}_{\Gamma}, \phi_{\Gamma}, \psi_{\Gamma}\right) .
$$

$\mathscr{L}_{\Gamma}\left(\mathscr{E}_{\Gamma}\right)$ is the set of internal (external) lines of $\Gamma$ and $\mathscr{B}_{\Gamma}$ is the set of vertices of $\Gamma$. Every internal line $L \in \mathscr{L}_{\Gamma}$ is mapped by $\phi_{\Gamma}$ to its endpoints $A_{L}, B_{L} \in \mathscr{B}_{\Gamma}: \phi_{\Gamma}(L)$ $=\left(A_{L}, B_{L}\right)$. Every external line $E \in \mathscr{E}_{\Gamma}$ is mapped by $\psi_{\Gamma}$ to its endpoint $B_{E}=\psi_{\Gamma}(E) \in \mathscr{B}_{\Gamma}$. The latter are called external vertices of $\Gamma$. A vertex is called an internal one if it is not an external vertex.

An external line $E \in \mathscr{E}_{\Gamma}$ carries an external momentum $q_{E}$ flowing into the diagram $\Gamma$. $q$ denotes a basis of the external momenta of $\Gamma$, e.g. $q=\left(q_{E_{1}}, \ldots, q_{E_{N-1}}\right)$, where $N$ is the number of external lines of $\Gamma . q_{E_{N}}$ is given by momentum conservation. Every internal line $L \in \mathscr{L}_{\Gamma}$ carries a momentum $l_{L}$ flowing from its outgoing endpoint $A_{L}$ to its ingoing endpoint $B_{L}$ and being a sum of the internal and external line momenta of $L[4]$

$$
l_{L}(k, q)=k_{L}(k)+q_{L}(q),
$$


where $(k)=\left(k_{1}, \ldots, k_{m}\right)$ is a basis of the internal (=loop) momenta of $\Gamma$. At every vertex, momentum conservation holds.

To every $L \in \mathscr{L}_{\Gamma}$ corresponds a propagator

$$
\widehat{\Delta}_{L}\left(l_{L} ; s, \mu, a\right)=\frac{\hat{P}_{L}\left(l_{L} ; s, \mu, a\right)}{\prod_{j=1}^{n(L)}\left[e_{L j}\left(l_{L} ; a\right)+(s-1)^{2} M_{L j}^{2}+\mu_{L j}^{2}\right]},
$$

where $n(L) \in \mathbb{N}$ and the auxiliary masses $M_{L j}$ are restricted by

$$
M_{L j}^{2}+\mu_{L j}^{2}>0 .
$$

Furthermore $^{1}, e_{L j} \in \mathscr{C}_{2}^{c}$, satisfying

$$
\begin{gathered}
e_{L j}\left(l_{L} ; a\right)=\frac{1}{a^{2}} \eta_{L j}\left(l_{L} a\right), \\
\eta_{L j}\left(l_{L} a \neq 0\right)>0 \text { if } l_{L} \in \mathrm{BZ}=[-\pi / a, \pi / a]^{4}, \\
\eta_{L j}\left(l_{L} a\right) \text { BZ-periodic in } l_{L}, \\
\lim _{a \rightarrow 0} e_{L j}\left(l_{L} ; a\right)=l_{L}^{2} .
\end{gathered}
$$

$s$ is the mass parameter mentioned in the introduction. The numerator is of the form

$$
\hat{P}_{L}\left(l_{L} ; s, \mu, a\right)=\sum_{(i)} P^{(i)}(\mu, s) V_{(i)}\left(l_{L} ; a\right),
$$

where the sum is finite, $P^{(i)}$ are polynomials and $V_{(i)} \in \mathscr{C}_{m_{t}}^{\mathrm{c}}$ BZ-periodic in $l_{L}, m_{i} \in \mathbb{Z}$. For every vertex $B \in \mathscr{B}_{\Gamma}$ we have a function

$$
\widehat{V}_{B}\left(\left\{l_{L}\right\}_{B} ; s, \mu, a\right) \in \mathscr{C}^{c}
$$

of a form (2.13) in variables $\left\{l_{L}\right\}_{B}$ which are the momenta of lines at the vertex $B . \hat{V}_{B}$ is always assumed to be periodic with the BZ in all momenta.

The unrenormalized Feynman integral of $\Gamma$ is given by

$$
\hat{\mathscr{I}}_{\Gamma}(q ; s, \mu, a)=\int_{-\pi / a}^{\pi / a} d^{4} k_{1}, \ldots, d^{4} k_{m} \hat{I}_{\Gamma}(k, q ; s, \mu, a),
$$

where $m$ is the number of loops in $\Gamma$ and

$$
\hat{I}_{\Gamma}(k, q ; s, \mu, a)=\prod_{B \in \mathscr{B}_{T}} \hat{V}_{B}\left(\left\{l_{L}\right\}_{B} ; s, \mu, a\right) \cdot \prod_{L \in \mathscr{\mathscr { L }}_{\Gamma}} \hat{\Delta}_{L}\left(l_{L} ; s, \mu, a\right) .
$$

This function belongs to the class of functions $\mathscr{F}$.

To define a renormalized Feynman integral we need a precise definition of internal and external momenta of $\Gamma$ as well as of every 1PI subdiagram $\gamma$ of $\Gamma: k^{\gamma}$, $q^{\gamma}$. This is done as in [4, Sect. 2.2]. In addition, in every propagator and vertex of $\gamma$ we have to substitute the mass parameter $s$ by $s^{\gamma}$. Correspondingly, the substitution operators of [4] must be generalized. For 1PI subdiagrams $\tau, \gamma$ of $\Gamma, \tau$ being a subdiagram of $\gamma$,

$$
S_{\gamma}: k^{\tau} \rightarrow k^{\tau}\left(k^{\gamma}\right), \quad q^{\tau} \rightarrow q^{\tau}\left(k^{\gamma}, q^{\gamma}\right), \quad s^{\tau} \rightarrow s^{\gamma},
$$

\footnotetext{
${ }^{1}$ The function classes $\mathscr{C}_{m}^{c}, \mathscr{C}^{c}$, and $\mathscr{F}$ used here are defined in [3] or repeatedly in [4, Appendix A]
} 
so that

$$
S_{\gamma} f\left(k^{\tau}, q^{\tau} ; s^{\tau}, \mu, a\right)=f\left(k^{\tau}\left(k^{\gamma}\right), q^{\tau}\left(k^{\gamma}, q^{\gamma}\right) ; s^{\gamma}, \mu, a\right) .
$$

When applied to $k^{\tau}, q^{\tau}, S_{\gamma}$ is defined as in [4]. We remind the reader that the $k^{\gamma}$ dependence of $q^{\tau}$ via $S_{\gamma}$ occurs only by the explicit $k^{\gamma}$-dependence of external lines of $\tau$, and that $k^{\tau}$ is independent of $q^{\gamma}$ via $S_{\gamma}$. Line momenta are always chosen in such a way that they are natural in the sense of [3].

The notion of a $\Gamma$-forest (set of non-trivial, non-overlapping 1PI subdiagrams of $\Gamma$ ) and related notions are defined in [5] or [4, Sect. 2], for instance. Especially, for any 1PI subdiagram $\gamma$ of $\Gamma$,

$$
U(\gamma) \equiv\left\{\gamma^{\prime} \in U \mid \gamma^{\prime} \text { is a subdiagram of } \gamma \text { and } \gamma^{\prime} \neq \gamma\right\} \text {, }
$$

and $\bar{\gamma}(U)=\gamma / \gamma_{1} \ldots \gamma_{c}$, where $\gamma_{1}, \ldots, \gamma_{c}$ are the maximal elements of $U(\gamma)$.

\subsection{Infrared and Ultraviolet Degrees}

As mentioned in the introduction, the auxiliary mass method implies that we also must differentiate a Feynman integrand with respect to the mass parameter $s$, to get convergence of the integral in the continuum limit. To describe the order of subtractions by divergence degrees, we have to introduce IR- and UV-degrees with respect to momentum and mass variables. We consider functions $V \in \mathscr{C}^{c}$ and $F \in \mathscr{F}$ of momentum variables $\left(u_{1}, \ldots, u_{r}\right),\left(v_{1}, \ldots, v_{d}\right),\left(q_{1}, \ldots, q_{w}\right),\left(\bar{q}_{1}, \ldots, \bar{q}_{z}\right)$ and of $s$ of the form

$$
V(u, v, q, \bar{q} ; s, \mu, a)=\sum_{i \in I} P_{i}(\mu, s) V_{i}(u, v, q, \bar{q} ; a),
$$

where $I$ is a finite set, $P_{i} \neq 0$ are polynomials and $V_{i} \in \mathscr{C}_{m_{i}}^{c}, m_{i} \in \mathbb{Z}, m_{i} \neq m_{k}$ if $i \neq k$, and

$$
F(u, v, q, \bar{q} ; s, \mu, a)=\frac{V(u, v, q, \bar{q} ; s, \mu, a)}{C(u, v, q, \bar{q} ; s, \mu, a)} .
$$

The numerator $V \in \mathscr{C}^{c}$ is assumed to be of the form (2.18),

$$
\begin{gathered}
C(u, v, q, \bar{q} ; s, \mu, a)=\prod_{i=1}^{n} C_{i}(u, v, q, \bar{q} ; s, \mu, a), \quad n \in \mathbb{N}_{0}=\{0,1,2, \ldots\}, \\
C_{i}(u, v, q, \bar{q} ; s, \mu, a)=e_{i}\left(l_{i} ; a\right)+(s-1)^{2} M_{i}^{2}+\mu_{i}^{2}, \quad e_{i} \in \mathscr{C}_{2}^{c} \text { of the form (2.12), } \\
l_{i}=\sum_{k=1}^{d} b_{i k} v_{k}+\sum_{k=1}^{r} c_{i k} u_{k}+\sum_{k=1}^{w} d_{i k} q_{k}+\sum_{k=1}^{z} e_{i k} \bar{q}_{k}, \\
\left(b_{i 1}, \ldots, b_{i d}\right) \neq 0 \text { or }\left(c_{i 1}, \ldots, c_{i r}\right) \neq 0 \text { for all } i=1, \ldots, n .
\end{gathered}
$$

Below $u$ will denote the parameters of a Zimmermann subspace $H$ [3], $v$ will be the complementary parameters, and $q, \bar{q}$ the external momenta of a diagram. $\bar{q}$ represents those external momenta appearing in the parametrization of $H$. IRdegrees are always defined for fixed $\bar{q}$. Non-fixed variables like $u, v, q, s-1$ are always explicitly indicated.

UV-degrees are defined as follows:

$$
\begin{aligned}
\overline{\operatorname{degr}}_{\hat{u q} s} V(u, v, q, \bar{q} ; s, \mu, a)= & \max _{i \in I}\left(\overline{\operatorname{degr}}_{s} P_{i}+\overline{\operatorname{degr}}_{\widehat{u q}} V_{i}\right), \\
\overline{\operatorname{degr}}_{\widehat{u} \hat{q} s} F(u, v, q, \bar{q} ; s, \mu, a) & =\overline{\operatorname{degr}}_{\hat{u q} s} V-\overline{\operatorname{degr}}_{\hat{u q} s} C(u, v, q, \bar{q} ; s, \mu, a) \\
& =\overline{\operatorname{degr}}_{\hat{u} \underline{q} s} V-2 n_{u q s},
\end{aligned}
$$


where $n_{u q s}$ is the number of factors $C_{i}$ depending on $u, q$ or $s$. IR-degrees are defined by

$$
\begin{gathered}
\underline{\operatorname{degr}}_{\hat{u q}(s-1) \mid v} V(u, v, q, \bar{q} ; s, \mu, a)=\min _{i \in I}\left(\underline{\operatorname{degr}} s-1 P_{i}+\underline{\operatorname{degr}_{\hat{u} q \mid v}} V_{i}\right), \\
\underline{\operatorname{degr}_{\hat{u} \mid v q(s-1)} V(u, v, q, \bar{q} ; s, \mu, a)=\min _{i \in I} \underline{\operatorname{degr}} \hat{u} \mid v q} V_{i}
\end{gathered}
$$

and

$$
\begin{aligned}
& \underline{\operatorname{degr}}_{\hat{u} q(s-1) \mid v} F(u, v, q, \bar{q} ; s, \mu, a)=\underline{\operatorname{degr}}_{\hat{u} q(s-1) \mid v} V(u, v, q, \bar{q} ; s, \mu, a)
\end{aligned}
$$

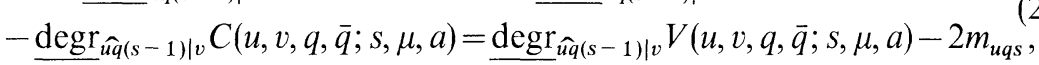

where $m_{u q s}$ is the number of $C_{i}$ which depend only on $u, q$ and $(s-1)($ i.e.

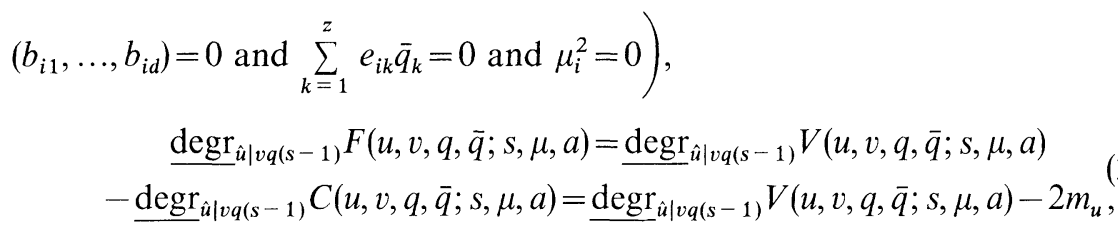

$m_{u}$ being the number of $C_{i}$ depending only on $u\left(\right.$ i.e. $\left(b_{i 1}, \ldots, b_{i d}\right)=0,\left(d_{i 1}, \ldots, d_{i w}\right)=0$, $\sum_{k=1}^{z} e_{i k} \bar{q}_{k}=0$ and $\left.\mu_{i}^{2}=M_{i}^{2}=0\right) \cdot \overline{\operatorname{degr}}_{s} P_{i}$ and $\underline{\operatorname{degr}}{ }_{s-1} P_{i}$ are the usual UV-and IRdegrees of polynomials (defined in [6, Appendix A], for instance).

The degrees satisfy all "typical degree properties." For completeness, they are listed in the appendix. Later on we will use them without any explicit reference.

We now define UV-and IR-divergence degrees of an arbitrary 1 PI subdiagram $\gamma$ of $\Gamma$ by

$$
\begin{gathered}
\omega(\gamma)=\sum_{L \in \mathscr{L}_{\gamma}} \omega\left(\hat{U}_{L}\right)+\sum_{B \in \mathscr{B}_{\gamma}} \omega\left(\hat{V}_{B}\right)+4 m(\gamma), \\
r(\gamma)=\sum_{L \in \mathscr{L}_{\gamma}} r\left(\hat{U}_{L}\right)+\sum_{B \in \mathscr{B}_{\gamma}} r\left(\hat{V}_{B}\right)+4 m(\gamma),
\end{gathered}
$$

where $m(\gamma)$ is the number of loops in $\gamma$, and

$$
\begin{aligned}
& \omega\left(\hat{U}_{L}\right)=\overline{\operatorname{degr}}_{\hat{l}_{L} s} \hat{\Delta}_{L}\left(l_{L} ; s, \mu, a\right), \\
& r\left(\hat{U}_{L}\right)=\underline{\operatorname{degr}} \hat{l}_{L}(s-1) \\
& \hat{\Delta}_{L}\left(l_{L} ; s, \mu, a\right)
\end{aligned}
$$

for $L \in \mathscr{L}_{\Gamma}$ and

$$
\begin{aligned}
\omega\left(\hat{V}_{B}\right) & =\overline{\operatorname{degr}}_{\left.\widehat{\{l}_{L}\right\}_{B} s} \hat{V}_{B}\left(\left\{l_{L}\right\}_{B} ; s, \mu, a\right), \\
r\left(\hat{V}_{B}\right) & =\underline{\operatorname{degr}_{\left\{l_{L}\right\}_{B}(s-1)} \hat{V}_{B}\left(\left\{l_{L}\right\}_{B} ; s, \mu, a\right)}
\end{aligned}
$$

for $B \in \mathscr{B}_{\Gamma}$. These definitions are valid also for reduced diagrams [4].

We will write the divergence degrees in a vertex dependent form. To every line $L \in \mathscr{L}_{\gamma}$ corresponds a pair of basic fields $A_{i}, A_{k}$. $L$ is called an $i k$-type line, having $i$-type and $k$-type legs. For every field $A_{i}$, a UV-dimension $d_{i}$ and an IR-dimension $r_{i}>0$ is defined such that

$$
4+\omega\left(\hat{\Delta}_{L}\right) \leqq d_{i}+d_{k}, \quad 4+r\left(\hat{\Delta}_{L}\right) \geqq r_{i}+r_{k} .
$$


Let $n_{k}(B)$ denote the number of $k$-type legs at the vertex $B \in \mathscr{B}_{\gamma}$ (including external legs) and $e_{k}(\gamma)$ the number of external $k$-type legs of $\gamma$. Then we can write

$$
\begin{gathered}
\omega(\gamma) \leqq \bar{\omega}(\gamma)=4+\sum_{B \in \mathscr{B}_{\gamma}}[\omega(B)-4]-\sum_{k} e_{k}(\gamma) d_{k}, \\
r(\gamma) \geqq \bar{r}(\gamma)=4+\sum_{B \in \mathscr{B}_{\gamma}}[r(B)-4]-\sum_{k} e_{k}(\gamma) r_{k},
\end{gathered}
$$

where

$$
\begin{gathered}
\omega(B)=\sum_{k} n_{k}(B) d_{k}+\omega\left(\hat{V}_{B}\right), \\
r(B)=\sum_{k} n_{k}(B) r_{k}+r\left(\hat{V}_{B}\right) .
\end{gathered}
$$

These forms of divergence degrees will be used in the following. Especially, we will see that the IR-divergence degrees $r(B)$ must satisfy some constraints to get IRconvergence of Feynman integrals.

A general statement about convergence of Feynman integrals with massless propagators can be made only for non-exceptional external momenta. The external momenta $q_{1}, \ldots, q_{N}$ of a 1 PI function

$$
\left\{\prod_{i=1}^{N} \widetilde{P}_{i}\left(q_{i}\right) \cdot \prod_{j=1}^{M}\left[Q_{j}\right]\right\}_{0,1 \mathrm{PI}}
$$

or of a contributing diagram $\Gamma$ are called non-exceptional [11] if

$$
\sum_{i=1}^{N} \alpha_{i} q_{i}=0, \quad \alpha_{i} \in\{0,1\}
$$

implies that all $\alpha_{i}=0$ or all $\alpha_{i}=1$.

\subsection{Generalized Subtraction Operators}

We now define generalized subtraction operators which apply to Feynman integrals with zero-mass propagators. Let $F$ be a function of the same momentum and mass variables as before which is $C^{\infty}$ in $q$ and $s$.

Definition 2.1. Let $\delta \in \mathbb{N}_{0}=\{0,1,2, \ldots\}$ and $\hat{t}_{q s}^{\gamma}$ be defined by

$$
\begin{gathered}
\left(\hat{t}_{q s}^{\delta} F\right)(u, v, q, \bar{q} ; s, \mu, a)=\sum_{0 \leqq b+g \leqq \delta} \frac{s^{b}}{b !} \frac{1}{g !} \sum_{i_{1}, \ldots, i_{g}=0}^{w} P_{g, i_{1}, \ldots, i_{g}}\left(q_{1}, \ldots, q_{w} ; a\right) \\
\times\left[\frac{\partial^{b}}{\partial s^{b}} \frac{\partial}{\partial q_{i_{1}}} \ldots \frac{\partial}{\partial q_{i_{g}}} F(u, v, q, \bar{q} ; s, \mu, a)\right]_{q=0, s=0}
\end{gathered}
$$

for every function $F$ which is $C^{\infty}$ in $q$ and $s$, where $P_{g, i_{1}, \ldots, i_{g}} \in \mathscr{C}_{g}^{c}$ are totally symmetric in $i_{1}, \ldots, i_{g},(2 \pi / a)$-periodic in $q_{1}, \ldots, q_{w}$, and $\lim _{a \rightarrow 0} P_{g, i_{1}, \ldots, i_{g}}\left(q_{1}, \ldots, q_{w} ; a\right)$ $=q_{i_{1}}, \ldots, q_{i_{g}}$. If for every such $F$

$$
\left[\left(1-\widehat{t}_{q s}^{o}\right) F\right](u, v, \lambda q, \bar{q} ; \lambda s, \mu, a)=O\left(\lambda^{\delta+1}\right) \quad \text { as } \quad \lambda \rightarrow 0
$$

$\widehat{t}_{q s}^{b}$ is called a generalized subtraction operator (GSO) of the order $\delta$. 
The generalization consists in that in $\hat{t}_{q s}^{\delta}$ one also differentiates with respect to the mass parameter $s$. By analogy, $\hat{t}_{q(s-1)}^{Q}$ is called a generalized subtraction operator of order $\varrho$, if for a function $F$ which is $C^{\infty}$ in $q$ and $s$ the function $\hat{t}_{q(s-1)}^{\varrho} F$ is of the form (2.33), where $\delta$ is replaced by $\varrho$ and $s$ by $(s-1)$, and

$$
\left[\left(1-\hat{t}_{q(s-1)}^{\varrho}\right) F\right](u, v, \lambda q, \bar{q} ; 1+\lambda(s-1), \mu, a)=O\left(\lambda^{\varrho+1}\right), \lambda \rightarrow 0 .
$$

Obviously, $\hat{t}_{q s}^{\widehat{x}}$ is a GSO of the order $\delta$ if $\hat{t}_{q}^{-b}$, defined by

$$
\begin{gathered}
\left(\hat{t}_{q}^{\delta-b} F\right)(u, v, q, \bar{q} ; s, \mu, a) \\
=\sum_{g=0}^{\delta-b} \frac{1}{g !} \sum_{i_{1}, \ldots, i_{g}=0}^{w} P_{g, i_{1}, \ldots, i_{g}}\left(q_{1}, \ldots, q_{w} ; a\right)\left(\frac{\partial}{\partial q_{i_{1}}} \ldots \frac{\partial}{\partial q_{i_{g}}} F(u, v, q, \bar{q} ; s, \mu, a)\right)_{q=0}
\end{gathered}
$$

is a subtraction operator [4] of the order $\delta-b$, for every $b$ satisfying $0 \leqq b \leqq \delta$. An analogous statement holds for $\hat{t}_{q(s-1)}^{o}$.

We want to apply GSO's to functions $F \in \mathscr{F}$ of the form (2.19). To this end, we have to exclude in (2.34) and (2.35) those values of the variables $u, v$ satisfying

$$
\sum_{k=1}^{d} b_{i k} v_{k}+\sum_{k=1}^{r} c_{i k} u_{k}+\sum_{k=1}^{z} e_{i k} \bar{q}_{k}=0
$$

for some $i \in\{1, \ldots, n\}$ with $\mu_{i}=0$.

Generalized subtraction operators have important properties which are responsible for the subtraction of UV-divergencies by applying them to Feynman integrals, and that subtracted diagrams are IR-finite. These properties will be given in Sect. 4 when we have defined the renormalization procedure.

\section{Renormalization of Lattice Green Functions}

We give a prescription how to renormalize 1PI lattice functions

$$
\left\{\prod_{i=1}^{N} \widetilde{P}_{i}\left(q_{i}\right) \cdot \prod_{j=1}^{M}\left[Q_{j}\right]\right\}_{0,1 \mathrm{PI}} .
$$

Expression (3.1) is a sum of 1PI Feynman integrals. At first, we define renormalized Feynman integrals. As indicated in the introduction and in Example 2.1, an important convergence condition is that every internal vertex $B$ must have an IRdivergence degree not less than four. This condition will be assumed in the following.

Let

$$
\Gamma=\left(\mathscr{L}_{\Gamma}, \mathscr{E}_{\Gamma}, \mathscr{B}_{\Gamma}, \phi_{\Gamma}, \psi_{\Gamma}\right)
$$

be a 1PI diagram with $m$ loops and $\hat{\mathscr{I}}_{\Gamma}(q ; s, \mu, a)$ the corresponding unrenormalized Feynman integral. The renormalized Feynman integral of $\Gamma$ is defined by

$$
\hat{\mathscr{R}}_{\Gamma}(q ; s, \mu, a)=\int_{-\pi / a}^{\pi / a} d^{4} k_{1}, \ldots, d^{4} k_{m} \hat{R}_{\Gamma}(k, q ; s, \mu, a),
$$

where

$$
\hat{R}_{\Gamma}(k, q ; s, \mu, a)=S_{\Gamma} \sum_{U \in \mathscr{W}} \prod_{\gamma \in U}\left(-\hat{\tau}_{\gamma} S_{\gamma}\right) \cdot \hat{I}_{\Gamma}(U)
$$


Here

1. $S_{\gamma}$ are the substitution operators (2.16).

2. $\mathscr{W}$ is the set of all $\Gamma$-forests.

3. $\hat{I}_{\Gamma}(U)$ is the unsubtracted Feynman integrand

$$
\hat{I}_{\Gamma}(k, q ; s, \mu, a)
$$

with the following substitutions depending on a forest $U$ :

For every line $L \in \mathscr{L}_{\Gamma}$ (vertex $B \in \mathscr{B}_{\Gamma}$ ) there is at most one $\gamma \in U$, so that $L \in \mathscr{L}_{\gamma}\left(B \in \mathscr{B}_{\gamma}\right)$, but $L \notin \mathscr{L}_{\gamma^{\prime}}\left(B \notin \mathscr{B}_{\gamma^{\prime}}\right)$ for all $\gamma^{\prime} \in U(\gamma)$. If such a $\gamma \in U$ exists, we write $\hat{\Delta}_{L}\left(\hat{V}_{B}\right)$ as a function of the variables $q^{\gamma}, k^{\gamma}, s^{\gamma}$, otherwise as a function of $k, q, s$.

4. $\hat{\tau}_{\gamma}$ is given by

$$
1-\hat{\tau}_{\gamma}=\left(1-\hat{t}_{q^{\gamma}\left(s^{\gamma}-1\right)}^{Q(\gamma)-1}\right)\left(1-\hat{t}_{q^{\gamma} s^{\gamma}}^{\delta(\gamma)}\right)
$$

for every 1PI subdiagram $\gamma$ of $\Gamma . \hat{t}_{q^{\gamma}\left(s^{\gamma}-1\right)}^{(\gamma)-1}$ and $\hat{t}_{q^{\gamma} s^{\gamma}}^{(\gamma)}$ are GSO's.

The UV-subtraction degrees $\delta(\gamma)$ and IR-subtraction degrees $\varrho(\gamma)$ are given by

$$
\begin{aligned}
& \delta(\gamma)=4+\sum_{B \in \mathscr{B}_{\gamma}}[\delta(B)-4]-\sum_{k} e_{k}(\gamma) d_{k}, \\
& \varrho(\gamma)=4+\sum_{B \in \mathscr{B}_{\gamma}}[\varrho(B)-4]-\sum_{k} e_{k}(\gamma) r_{k} .
\end{aligned}
$$

$e_{k}(\gamma)$ is the number of external $k$-type legs of $\gamma \cdot r_{k}>0$ and $d_{k}$ are the IR-and UV-dimensions of the field $A_{k}$ [cf. (2.29)]. Furthermore, the UV-and IR-subtraction degrees $\delta(B)$ and $\varrho(B), B \in \mathscr{B}_{\Gamma}$, are constrained by

$$
\begin{array}{ll}
\quad \delta(B) \geqq \omega(B) & \text { for every vertex } B \in \mathscr{B}_{\Gamma}, \\
\varrho(B) \leqq \delta(B) & \\
\varrho(B)=4 & \text { for every internal vertex } B \in \mathscr{B}_{\Gamma} \\
\varrho(B) \leqq \min (4, r(B)) & \text { for every external vertex } B \in \mathscr{B}_{\Gamma} .
\end{array}
$$

$\omega(B)$ and $r(B)$ are the UV- and IR-divergence degrees of the vertex $B$ [cf. (2.31)]. Note that always $\varrho(\gamma)-1 \leqq \delta(\gamma)$. If $\delta(\gamma)<0$, we set $\hat{t}_{q^{\gamma} s^{\gamma}}^{\gamma(\gamma)}=0$, and if $\varrho(\gamma)-1<0$ : $\hat{t}_{q^{\gamma}\left(s^{\gamma}-1\right)}^{o(\gamma)-1}=0$.

5. The order of the factors in

$$
\prod_{\gamma \in U}\left(-\hat{\tau}_{\gamma} S_{\gamma}\right)
$$

is determined by the rule that for $\gamma_{1}, \gamma_{2} \in U, \gamma_{1}$ being a subdiagram of $\gamma_{2}$

$$
\left(-\hat{\tau}_{\gamma_{1}} S_{\gamma_{1}}\right) \text { is ordered to the right of }\left(-\hat{\tau}_{\gamma_{2}} S_{\gamma_{2}}\right) \text {. }
$$

For disjoint $\gamma_{1}, \gamma_{2}$, the order is irrelevant.

Theorem 1. Assume that every internal vertex $B$ of the diagram $\Gamma$ satisfies $r(B) \geqq 4$, and that the external momenta of $\Gamma$ are non-exceptional. Then the renormalized Feynman integral $\widehat{\mathscr{R}}_{\Gamma}(q ; s, \mu, a)$ is absolutely convergent for every $s$ and $a>0$. The continuum limit $a \rightarrow 0$ exists and is given $b y$

$$
\lim _{a \rightarrow 0} \widehat{\mathscr{R}}_{\Gamma}(q ; s, \mu, a)=\int_{-\infty}^{\infty} d^{4} k_{1}, \ldots, d^{4} k_{m} R_{I}(k, q, s, \mu),
$$


where

$$
R_{\Gamma}(k, q, s, \mu)=\lim _{a \rightarrow 0} \hat{R}_{\Gamma}(k, q ; s, \mu, a) .
$$

If $\lim _{a \rightarrow 0} \hat{I}_{\Gamma}(k, q ; s, \mu, a) \neq 0, R_{\Gamma}$ is equal to the BPHZL renormalized continuum Feynman integrand defined in [10] (with a different choice of internal momenta [4]). If $\lim _{a \rightarrow 0} \hat{I}_{\Gamma}(k, q ; s, \mu, a) \equiv 0$, also $R_{\Gamma}(k, q, s, \mu) \equiv 0$. As for massive field theories, Feynman integrals which have a vertex with vanishing (naive) continuum limit do not contribute to the continuum limit at all, after renormalization.

Renormalized diagrams are convergent also for $s=1$. As an important convergence condition, $r(B) \geqq 4$ for all internal vertices $B$ of the diagram, i.e. those vertices having no external line. In general, they result from an interaction or counterterm contribution to the lattice action. As the theorem shows, vertices with an external line do not have to satisfy such a constraint. This means that the (nonvanishing) external momenta provide an IR-cutoff. In most cases, the IRsubtraction degrees satisfy $\varrho(\gamma) \geqq 1$ only for diagrams $\gamma$ with two or three massless external lines, so that these are the only Feynman graphs which are affected by the additional finite renormalizations [cf. (3.5), (3.7)]. After renormalization, they vanish at zero external momenta.

As a corollary of this theorem we state the renormalization prescription for 1PI functions (3.1). Such a Green function is a finite sum of 1PI Feynman diagrams. The renormalization prescription is as follows. Every contributing diagram $\gamma$ will be renormalized as described by Theorem 1 . The subtraction degrees are given by (3.8), (3.9). These conditions, however, do not completely fix the subtraction degrees of the vertices. This is done in the following way. $P_{i}$ may be a basic field or a composite operator. In the latter case, there corresponds an external vertex to $\widetilde{P}_{i}$, in every diagram $\gamma$ which contributes to (3.1). Furthermore, to every $Q_{j}$ corresponds a vertex in every $\gamma$ which may be an internal or an external one. For every composite $\widetilde{P}_{i}$ and for every $Q_{j}$ we denote the UV-divergence degree of the corresponding vertex by $\omega_{i}$ and $\tau_{j}$ and the IR-divergence degrees by $r_{i}$ and $v_{j}$, respectively. These numbers are independent of $\gamma$ and depend only on the form of $\widetilde{P}_{i}$ and $Q_{j}$. In the same way, let for every $\widetilde{P}_{i}$ and $Q_{j} \mathrm{UV}$-subtraction degrees of the vertices be given by $\delta_{i}$ and $\eta_{j}$, and IR-subtraction degrees by $\varrho_{i}$ and $\sigma_{j}$, respectively. They are always chosen to be the same for all diagrams $\gamma$ contributing to (3.1). Furthermore, they are constrained by the conditions

$$
\begin{gathered}
\delta_{i} \geqq \omega_{i}, \quad \varrho_{i} \leqq \min \left(4, r_{i}, \delta_{i}\right) \quad \text { for composite } P_{i} \\
\eta_{j} \geqq \max \left(4, \tau_{j}\right), \quad \sigma_{j}=4, \quad \text { for all } j=1, \ldots, M .
\end{gathered}
$$

In general, the constraints (3.8), (3.9) are satisfied for every contributing diagram if (3.11), (3.12) hold.

Let $P_{1}, \ldots, P_{N_{0}}$ be basic fields and $P_{N_{0}+1}, \ldots, P_{N}$ be composite operators. Then the renormalized Green function of (3.1) is written as

$$
\left\{\prod_{i=1}^{N_{0}} \widetilde{P}_{i}\left(q_{i}\right) \cdot \prod_{N_{0}+1}^{N} N_{\delta_{i}}^{Q_{i}}\left[\widetilde{P}_{i}\left(q_{i}\right)\right] \cdot \prod_{j=1}^{M}\left[Q_{j}\right]_{\eta_{j}}^{\sigma_{J}}\right\}_{0,1 \mathrm{PI}},
$$

and we can state the following 
Theorem 2. The 1PI function (3.13) is finite for each $a>0$, and also in the continuum limit $a \rightarrow 0$, if the following conditions are satisfied.

1. The external momenta $q_{1}, \ldots, q_{N}$ are non-exceptional.

2. $v_{j} \geqq 4$ for all $j=1, \ldots, M$.

The $a \rightarrow 0$-limit is given by the BPHZL renormalized continuum Green function of (3.1) [10].

From Theorem 2 we easily get a renormalization prescription for vertex functions. They are not necessarily 1PI, e.g. for theories with spontaneously broken symmetries. Nevertheless, every diagram which contributes to a vertex function is a product of 1PI diagrams and other, finite terms. If the 1PI graphs are renormalized as described above and such that renormalized tadpole diagrams vanish, the renormalized vertex functions are IR-finite and convergent in the continuum limit. Every tadpole line entering a 1PI subgraph is an external line of this subdiagram of vanishing momentum, hence could produce an IR-singularity. Vanishing renormalized one-point functions prevent such IR-divergencies.

When Ward-identities are to be satisfied by the vertex functions, then in general additional finite renormalizations of 1PI functions are necessary. This can lead to non-vanishing one-point functions. In this case, it must be checked very carefully whether no IR-singularities are produced. In particular, every vertex $V$ with a leg which gives rise to tadpoles must satisfy stronger IR-constraints than $r(V) \geqq 4$, namely, omitting the tadpole line, the resulting vertex $V^{\prime}$ also should satisfy the condition $r\left(V^{\prime}\right) \geqq 4$.

The above theorem gives a well-defined procedure to renormalize theories containing massless fields $(s=1)$. Massless bare fields remain massless after renormalization. With respect to universality and power counting renormalizability, the same arguments as in [4] go through. The same holds for the counterterm philosophy. All subtractions can be written as counterterms of the lattice action, eventually after some symmetrizations of the subtractions [4]. Also, they can always be chosen to be local. Counterterms and consequently renormalized Green functions depend on the auxiliary masses. This dependence can be absorbed by addition of finite counterterms satisfying the IR-constraints. In this way, normalization conditions at non-exceptional momenta may be implemented.

The constraints on the IR-subtraction degrees are stronger than those of [10]. This is not a lattice artifact. They are necessary to avoid IR-singularities by subtractions. When we consider lattice Green functions in configuration space, the external momenta are integrated over, and the constraints (3.11), (3.12) may be replaced by the weaker conditions

$$
\begin{gathered}
\delta_{i} \geqq \omega_{i}, \quad \varrho_{i} \leqq \min \left(r_{i}, \delta_{i}\right), \\
\eta_{j} \geqq \tau_{j}, \quad 4 \leqq \sigma_{j} \leqq \min \left(v_{j}, \eta_{j}\right) .
\end{gathered}
$$

As a simple example of Theorem 2, consider the massless lattice $\Phi^{4}$-theory with an additional $\Phi^{6}$-interaction:

$$
S(\Phi)_{\mathrm{int}}=a^{4} \sum_{n \in \mathbb{Z}^{4}}\left[g \Phi^{4}(n a)+\lambda a^{2} \Phi^{6}(n a)\right] .
$$


The IR-dimension of the $\Phi$-field is equal to one. Hence, $r\left(\Phi^{4}\right)=4$ and $r\left(a^{2} \Phi^{6}\right)=6$. Powers of the lattice spacing have no influence on the IR-degrees. Hence, the massless model is IR-finite renormalizable.

\section{Properties of Generalized Subtraction Operators}

Before we are going to prove Theorem 1, we list important properties of GSO's. The first two lemmas state those properties of GSO's which are responsible for the subtraction of UV-divergencies by applying them to Feynman integrals as described in Sect. 3.

Lemma 4.1. Let $\widehat{t}_{q s}^{\mathrm{s}}$ and $\hat{t}_{q(s-1)}^{\mathrm{Q}}$ be GSO's and $F \in \mathscr{F}$ of the form (2.19). Then

$$
\begin{aligned}
& \text { 1. a) } \overline{\operatorname{degr}}_{\hat{v}} \hat{t}_{q(s-1)}^{o} F \leqq \overline{\operatorname{degr}}_{\hat{v}} F, \\
& \text { b) } \overline{\operatorname{degr}}_{\hat{v}} \hat{t}_{q s}^{o} F \leqq \overline{\operatorname{degr}}_{\hat{v}} F .
\end{aligned}
$$

Suppose that for every $i=1, \ldots, n$ the coefficients satisfy $\left(b_{i 1}, \ldots, b_{i d}\right)=0$ only if $\left(d_{i 1}, \ldots, d_{i w}\right)=0$ and $M_{i}^{2}=0$. Then

$$
\begin{aligned}
& \text { 2. a) } \overline{\operatorname{degr}}_{\hat{v} \hat{q} s} \hat{t}_{q(s-1)}^{\varrho} F \leqq \overline{\operatorname{degr}}_{\hat{v} q s} F, \\
& \text { b) } \overline{\operatorname{degr}}_{\hat{v} q s} \hat{t}_{q s}^{\delta} F \leqq \overline{\operatorname{degr}}_{v \hat{q} s} F .
\end{aligned}
$$

Proof.

$$
\begin{gathered}
\overline{\operatorname{degr}}_{\hat{v}}\left[\frac{\partial^{b}}{\partial(s-1)^{b}} \frac{\partial^{l}}{\partial q^{l}} F(u, v, q, \bar{q} ; s, \mu, a)\right]_{q=0, s-1=0} \\
\leqq \overline{\operatorname{degr}}_{\hat{v}} \frac{\partial^{b}}{\partial(s-1)^{b}} \frac{\partial^{l}}{\partial q^{l}} F(u, v, q, \bar{q} ; s, \mu, a) \leqq \overline{\operatorname{degr}}_{\hat{v}} F(u, v, q, \bar{q} ; s, \mu, a),
\end{gathered}
$$

and that proves 1.a. The proof of 1.b follows the same way. To prove 2.a, note that by assumption all propagators which depend on $q$ or $(s-1)$ are also dependent on $v$, hence

$$
\begin{gathered}
\overline{\operatorname{degr}}_{\hat{v q} s}\left[\frac{\partial^{b}}{\partial(s-1)^{b}} \frac{\partial^{l}}{\partial q^{l}} F(u, v, q, \bar{q} ; s, \mu, a)\right]_{q=0, s-1=0} \\
\leqq \overline{\operatorname{degr}}_{\hat{v q s} s} \frac{\partial^{b}}{\partial(s-1)^{b}} \frac{\partial^{l}}{\partial q^{l}} F(u, v, q, \bar{q} ; s, \mu, a) \leqq \overline{\operatorname{degr}}_{\hat{v q} s} F(u, v, q, \bar{q} ; s, \mu, a)-(b+|l|),
\end{gathered}
$$

where $|l|=\sum_{i=1}^{w} l_{i}$. Consequently

$$
\begin{gathered}
\overline{\operatorname{degr}}_{\hat{v} q s}(s-1)^{b} P_{g, i_{1}, \ldots, i_{g}}\left(q_{1}, \ldots, q_{w} ; a\right)\left[\frac{\partial^{b}}{\partial(s-1)^{b}} \frac{\partial^{l}}{\partial q^{l}} F(u, v, q, \bar{q} ; s, \mu, a)\right]_{q=0, s-1=0} \\
\leqq(g+b)+\overline{\operatorname{degr}}_{\hat{v} q s} F(u, v, q, \bar{q} ; s, \mu, a)-(b+|l|)=\overline{\operatorname{degr}}_{\hat{v} q s} F(u, v, q, \bar{q} ; s, \mu, a),
\end{gathered}
$$

for $|l|=g .2 . b$ follows analogously.

Lemma 4.2. Let $\hat{t}_{q(s-1)}^{o-1}, \widehat{t}_{q s}^{\delta}$ GSO's, $\varrho-1 \leqq \delta$, and $\hat{\tau}_{q s}^{o \delta}: \mathscr{F} \rightarrow \mathscr{F}$ defined $b y$

$$
1-\hat{\tau}_{q s}^{\varrho \delta}=\left(1-\hat{t}_{q(s-1)}^{\varrho-1}\right)\left(1-\hat{t}_{q s}^{\delta}\right) .
$$


Let $F \in \mathscr{F}$ be of the form (2.19). Then

$$
\begin{aligned}
& \text { 1. } \overline{\operatorname{degr}}_{\hat{v} \hat{q} s} \hat{\tau}_{q s}^{\varrho \delta} F \leqq \overline{\operatorname{degr}}_{\hat{v}} \hat{\tau}_{q S}^{\varrho \delta} F+\delta . \\
& \text { 2. } \overline{\operatorname{degr}}_{\hat{v}} \hat{\tau}_{q S}^{\varrho \delta} F \leqq \overline{\operatorname{degr}}_{\hat{v}} F .
\end{aligned}
$$

Assume that for every $i=1, \ldots, n$ the coefficients satisfy $\left(b_{i 1}, \ldots, b_{i d}\right)=0$ only if $\left(d_{i 1}, \ldots, d_{i w}\right)=0$ and $M_{i}^{2}=0$. Then

$$
\text { 3. } \overline{\operatorname{degr}}_{\hat{v q s}} \hat{\tau}_{q s}^{\varrho \delta} F \leqq \overline{\operatorname{degr}}_{\hat{v q s}} F \text {, }
$$

and

$$
\text { 4. } \overline{\operatorname{degr}}_{\hat{v}}\left(1-\hat{\tau}_{q s}^{\varrho \delta}\right) F \leqq \overline{\operatorname{degr}}_{\hat{v} s} F-(\delta+1) .
$$

These are exactly the properties necessary to reduce UV-divergence degrees of a Feynman integral systematically by application of $\hat{\tau}_{q s}^{\varrho \delta}$. The statements 2 . and 3 . are direct consequences of Lemma 4.1. We only have to note that if $F$ satisfies the additional constraint so does $\hat{t}_{q s}^{\gamma} F$. The proof of 1 . is nearly identical to that of $[4$, Lemma 3.1.1]. To prove 4., note that

$$
\overline{\operatorname{degr}}_{\hat{v}}\left(1-\hat{\tau}_{q s}^{\varrho \delta}\right) F(u, v, q, \bar{q} ; s, \mu, a) \leqq \overline{\operatorname{degr}}_{\hat{v}}\left(1-\widehat{t}_{q s}^{\delta}\right) F,
$$

by Lemma 4.1. Hence it is sufficient to show that

$$
\overline{\operatorname{degr}}_{\hat{v}}\left(1-\widehat{t}_{q s}^{\delta}\right) F \leqq \overline{\operatorname{degr}}_{\hat{v} \hat{q} s} F-(\delta+1),
$$

and this is done by using the same methods as in [4, Lemma 3.1.4].

The following two lemmas state properties of GSO's with respect to the IRdegrees of a function. Note that the $\bar{q}$ are fixed momenta.

Lemma 4.3. Let $\hat{t}_{q s}^{\delta}$ be $a \mathrm{GSO}$ and $F \in \mathscr{F}$ of the form (2.19). If for all $i=1, \ldots, n$ the coefficients satisfy $\left(d_{i 1}, \ldots, d_{i w}\right) \neq 0$ only if $M_{i}^{2}+\mu_{i}^{2} \neq 0$, the inequality

$$
\underline{\operatorname{degr}}_{\hat{u} \mid q v(s-1)} \hat{t}_{q s}^{\delta} F \geqq \underline{\operatorname{degr}_{\hat{u} \mid q v(s-1)} F}
$$

holds.

Proof. At first, we have

$$
\begin{gathered}
\operatorname{degr}_{\hat{u} \mid q v(s-1)}\left[\frac{\partial^{b}}{\partial s^{b}} \frac{\partial^{l}}{\partial q^{l}} F(u, v, q, \bar{q} ; s, \mu, a)\right]_{q=0, s=0} \\
\geqq \operatorname{degr}_{\hat{u} \mid q v(s-1)} \frac{\partial^{b}}{\partial s^{b}} \frac{\partial^{l}}{\partial q^{l}} F(u, v, q, \bar{q} ; s, \mu, a) \geqq \underline{\operatorname{degr}}_{\hat{u} \mid q v(s-1)} F(u, v, q, \bar{q} ; s, \mu, a),
\end{gathered}
$$

where for the first inequality we have used the constraints on the denominator of $F$. Relation (4.8) is now a direct consequence of the inequality.

Lemma 4.4. Let $\hat{t}_{q(s-1)}^{Q}$ be $a \mathrm{GSO}$ and $F \in \mathscr{F}$ of the form (2.19). Then

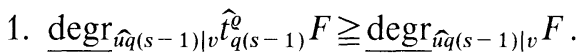

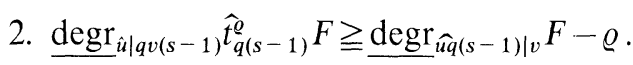


If for every $i=1, \ldots, n$ the coefficients satisfy $\left(b_{i 1}, \ldots, b_{i d}\right)=0$ only if $\left(d_{i 1}, \ldots, d_{i w}\right)=0$ and $M_{i}^{2}=0$, the inequalities

$$
\text { 3. } \underline{\operatorname{degr}}_{\hat{u} \mid q v(s-1)} \hat{t}_{q(s-1)}^{\underline{q}} F \geqq \underline{\operatorname{degr}}_{\hat{u} \mid q v(s-1)} F \text {, }
$$

and

$$
\text { 4. } \underline{\operatorname{degr}}_{\hat{u} \hat{q}(s-1) \mid v}\left(1-\hat{t}_{q(s-1)}^{Q}\right) F \geqq \overline{\operatorname{degr}}_{\hat{u} \mid q v(s-1)} F+\max (0, \varrho+1)
$$

hold.

Proof. Let $b \in \mathbb{N}_{0}$ and $l=\left(l_{1}, \ldots, l_{w}\right) \in \mathbb{N}_{0}^{w}$.

1. For every function $F \in \mathscr{F}$, we have

$$
\begin{aligned}
& \underline{\operatorname{degr}}_{\hat{u} \hat{q}(s-1) \mid v}\left[\frac{\partial^{b}}{\partial(s-1)^{b}} \frac{\partial^{l}}{\partial q^{l}} F(u, v, q, \bar{q} ; s, \mu, a)\right]_{q=0, s-1=0} \\
& \quad \geqq \underline{\operatorname{degr}}_{\hat{u} \hat{q}(s-1) \mid v} \frac{\partial^{b}}{\partial(s-1)^{b}} \frac{\partial^{l}}{\partial q^{l}} F(u, v, q, \bar{q} ; s, \mu, a) \\
& \geqq \underline{\operatorname{degr}}_{\hat{u} q(s-1) \mid v} F(u, v, q, \bar{q} ; s, \mu, a)-(b+|l|),
\end{aligned}
$$

hence for $g=|l|$

$$
\begin{aligned}
& \underline{\operatorname{degr}}_{\widehat{u} \vec{q}(s-1) \mid v}(s-1)^{b} P_{g, i_{1}, \ldots, i_{g}}\left(q_{1}, \ldots, q_{w} ; a\right)\left[\frac{\partial^{b}}{\partial(s-1)^{b}} \frac{\partial^{l}}{\partial q^{l}} F(u, v, q, \bar{q} ; s, \mu, a)\right]_{q=0, s-1=0} \\
& \geqq(b+g)+\underline{\operatorname{deg}}_{\hat{u} q(s-1) \mid v} F(u, v, q, \bar{q} ; s, \mu, a)-(b+|l|) \\
& =\underline{\operatorname{deg}}_{\hat{u} \hat{q}(s-1) \mid v} F(u, v, q, \bar{q} ; s, \mu, a) \text {. }
\end{aligned}
$$

The first statement of the lemma is now a direct consequence of this inequality.

2. For every $F \in \mathscr{F}$, we have

$$
\begin{gathered}
\underline{\operatorname{degr}}_{\hat{u} \mid q v(s-1)}\left[\frac{\partial^{b}}{\partial(s-1)^{b}} \frac{\partial^{l}}{\partial q^{l}} F(u, v, q, \bar{q} ; s, \mu, a)\right]_{q=0, s-1=0} \\
\geqq \underline{\operatorname{degr}_{\hat{u}(s-1) \mid v}} \frac{\partial^{b}}{\partial(s-1)^{b}} \frac{\partial^{l}}{\partial q^{l}} F(u, v, q, \bar{q} ; s, \mu, a) \\
\geqq \underline{\operatorname{degr}}_{\widehat{u} \hat{q}(s-1) \mid v} F(u, v, q, \bar{q} ; s, \mu, a)-(b+|l|),
\end{gathered}
$$

and for $g=|l|$

$$
\begin{aligned}
& \underbrace{}_{\operatorname{deg} r_{\hat{u} \mid q v(s-1)}(s-1)^{b}} P_{g, i_{1}, \ldots, i_{g}}\left(q_{1}, \ldots, q_{w} ; a\right)\left[\frac{\partial^{b}}{\partial(s-1)^{b}} \frac{\partial^{l}}{\partial q^{l}} F(u, v, q, \bar{q} ; s, \mu, a)\right]_{q=0, s-1=0} \\
& \geqq \underline{\operatorname{degr}}_{\widehat{u} \hat{q}(s-1) \mid v} F(u, v, q, \bar{q} ; s, \mu, a)-(b+g),
\end{aligned}
$$

i.e.

$$
\underline{\operatorname{degr}}_{\hat{u} \mid q v(s-1)} \hat{t}_{q(s-1)}^{\hat{Q}} F \geqq \underline{\operatorname{degr}}_{\hat{u} q(s-1) \mid v} F-\varrho .
$$

This proves the second statement of the lemma. 
3. All the propagators which depend on $q$ or have a non-vanishing auxiliary mass are also dependent on $v$. Hence

$$
\begin{aligned}
\operatorname{degr}_{\hat{u} \mid q v(s-1)}\left[\frac{\partial^{b}}{\partial(s-1)^{b}} \frac{\partial^{l}}{\partial q^{l}} F(u, v, q, \bar{q} ; s, \mu, a)\right]_{q=0, s-1=0} \\
\geqq \underline{\operatorname{degr}}_{\hat{u} \mid q v(s-1)} \frac{\partial^{b}}{\partial(s-1)^{b}} \frac{\partial^{l}}{\partial q^{l}} F(u, v, q, \bar{q} ; s, \mu, a) \\
\geqq \underline{\operatorname{degr}}_{\hat{u} \mid q v(s-1)} F(u, v, q, \bar{q} ; s, \mu, a) .
\end{aligned}
$$

Consequently

$$
\begin{gathered}
\operatorname{degr}_{\hat{u} \mid q v(s-1)}(s-1)^{b} P_{g, i_{1}, \ldots, i_{\mathrm{g}}}\left(q_{1}, \ldots, q_{w} ; a\right)\left[\frac{\partial^{b}}{\partial(s-1)^{b}} \frac{\partial^{l}}{\partial q^{l}} F(u, v, q, \bar{q} ; s, \mu, a)\right]_{q=0, s-1=0} \\
\geqq \underline{\operatorname{degr}}_{\hat{u} \mid q v(s-1)} F(u, v, q, \bar{q} ; s, \mu, a),
\end{gathered}
$$

and this proves the third inequality.

4. $\left(1-\hat{t}_{q(s-1)}^{\varrho}\right) F$ is of the form

$$
\left[\left(1-\hat{t}_{q(s-1)}^{\varrho}\right) F\right](u, v, q, \bar{q} ; s, \mu, a)=\frac{V_{\varrho}(u, v, q, \bar{q} ; s, \mu, a)}{C(u, v, q, \bar{q} ; s, \mu, a) C(u, v, 0, \bar{q} ; 1, \mu, a)^{\varrho+1}},
$$

where $V_{\varrho} \in \mathscr{C}^{c}$. Using the behavior (2.35) of the subtracted function and that $V_{\varrho} \in C^{\infty}$, we get

$$
V_{\varrho}(u, v, \lambda q, \bar{q} ; 1+\lambda(s-1), \mu, a)=O\left(\lambda^{\varrho+1}\right), \lambda \rightarrow 0 .
$$

This yields

$$
\underline{\operatorname{degr}}_{\hat{u} \hat{q}(s-1) \mid v} V_{\varrho} \geqq \underline{\operatorname{degr}}_{\hat{u} \mid q v(s-1)} V_{\varrho}+\max (0, \varrho+1) .
$$

By the constraint on the denominator, we get

$$
\underline{\operatorname{degr}}_{\hat{u} q(s-1) \mid v} C(u, v, q, \bar{q} ; s, \mu, a)=\underline{\operatorname{degr}}_{\hat{u} \mid q v(s-1)} C(u, v, q, \bar{q} ; s, \mu, a),
$$

hence

$$
\begin{aligned}
& \operatorname{degr}_{\widehat{u} \hat{q}(s-1) \mid v}\left[\left(1-\hat{t}_{q(s-1)}^{\varrho}\right) F\right](u, v, q, \bar{q} ; s, \mu, a) \\
& \quad \geqq \operatorname{degr}_{\hat{u} \mid q v(s-1)} V_{\varrho}(u, v, q, \bar{q} ; s, \mu, a) \\
& \quad-\underline{\operatorname{degr}}_{\hat{u} \mid q v(s-1)}\left[C(u, v, q, \bar{q} ; s, \mu, a) \cdot C(u, v, 0, \bar{q} ; 1, \mu, a)^{\varrho+1}\right]+\max (0, \varrho+1) \\
& \quad=\underline{\operatorname{degr}}_{\hat{u} \mid q v(s-1)}\left(1-\hat{t}_{q(s-1)}^{\varrho}\right) F+\max (0, \varrho+1) \\
& \quad \geqq \underline{\operatorname{degr}}_{\hat{u} \mid q v(s-1)} F+\max (0, \varrho+1),
\end{aligned}
$$

where we have used Lemma 4.4.3.

\section{Convergence Proof}

To prove Theorem 1 we show that all conditions to apply the power counting theorem of [6] to (3.2) are satisfied. Let $\Gamma$ be a 1 PI diagram and $m$ the number of loops in $\Gamma$. The subtracted Feynman integrand (3.3) corresponding to $\Gamma$ is of the form

$$
\hat{R}_{\Gamma}(k, q ; s, \mu, a)=\frac{V(k, q ; s, \mu, a)}{B_{1}(k, q ; s, \mu, a) B_{2}(k ; \mu, a)},
$$


where

$$
\begin{gathered}
B_{1}(k, q ; s, \mu, a)=\prod_{L \in \mathscr{L}_{\Gamma}} \prod_{j=1}^{n(L)}\left(e_{L j}\left(l_{L} ; a\right)+(s-1)^{2} M_{L j}^{2}+\mu_{L j}^{2}\right) \\
B_{2}(k ; \mu, a)=\prod_{\gamma} \prod_{L \in \mathscr{L}_{\gamma}} \prod_{j=1}^{n(L)}\left[e_{L j}\left(k_{L}^{\gamma} ; a\right)+M_{L j}^{2}+\mu_{L j}^{2}\right]^{n_{1 j}(L, \gamma)}\left[e_{L j}\left(k_{L}^{\gamma} ; a\right)+\mu_{L j}^{2}\right]^{n_{2 J}(L, \gamma)}
\end{gathered}
$$

$n_{1 j}(L, \gamma), n_{2 j}(L, \gamma) \in\{0,1,2, \ldots\}$, the first product is over all 1PI subdiagrams $\gamma$ of $\Gamma$, and

$$
l_{L}(k, q)=k_{L}(k)+q_{L}(q), \quad k_{L}^{\gamma}=k_{L}^{\gamma}(k) .
$$

$\hat{R}_{\Gamma}$ belongs to the class of functions $\mathscr{F}$ and is periodic in $k_{1}, \ldots, k_{m}$.

Let $\mathscr{L}$ be the set of all $l_{L}, L \in \mathscr{L}_{\Gamma}$, and of all $k_{L}^{\gamma}$ for arbitrary 1 PI subdiagrams $\gamma$ of $\Gamma$ and $L \in \mathscr{L}_{\gamma}$. The set $\mathscr{L}$ is natural [4].

Let

$$
u_{1}, \ldots, u_{r}, \quad v_{1}, \ldots, v_{d}
$$

be an arbitrary basis of $\mathscr{L}, r+d=m\left(u_{1}, \ldots, u_{r}, v_{1}, \ldots, v_{d} \in \mathscr{L}\right.$ and $\operatorname{det}(\partial(u, v) / \partial(k))$ $\neq 0$ ), and let $H$ be a Zimmermann subspace, i.e. a class of affine subspaces of $\left(k_{1}, \ldots, k_{m}\right)$, defined by constant $u_{1}, \ldots, u_{r}$ and variable $v_{1}, \ldots, v_{d}$. Then all $k, k^{\gamma}$ are linear functions in $u, v, q$ :

$$
k=k(u, v, q), \quad k^{\gamma}=k^{\gamma}(u, v, q) .
$$

$(v)=\left(v_{1}, \ldots, v_{d}\right)$ is called the parametrization of $H$. The set of all classes $H$, for all bases (5.4), is denoted by $\mathscr{H}$.

We will show that for every $H \in \mathscr{H}$

$$
\begin{aligned}
& 4 d+\overline{\operatorname{degr}}_{\hat{v}} \hat{R}_{\Gamma}(k(u, v, q), q ; s, \mu, a)<0, \quad \text { if } \quad d>0, \\
& 4 r+\left.\underline{\operatorname{degr}}_{\hat{u} \mid v} \hat{R}_{\Gamma}(k(u, v, q), q ; s, \mu, a)\right|_{s=1}>0, \quad \text { if } \quad r>0 \text {. }
\end{aligned}
$$

Then all the conditions are met for the power counting theorem of [6] to apply to the renormalized Feynman integral (3.2), and Theorem 1 is proved. Note that (II) must only be shown for $s=1$. If $s \neq 1$, all propagators are massive and (II) is trivially satisfied.

To prove (I) and (II) we will use the method of complete forests [5]. $\hat{R}_{\Gamma}(k, q ; s, \mu, a)$ is written in a form which depends on $H$, i.e. as a sum of terms which are described by complete forests and satisfy (I) and (II). A $\Gamma$-forest $U \in \mathscr{W}$ is called complete on $H$, parametrized by $(v)$, if $\Gamma \in U$, and if for any $\gamma \in U$ all lines of $\bar{\gamma}(U)$ are constant on $H$ relative to $\gamma$, i.e.

$$
k_{L}^{\gamma}(u, v, q) \text { is independent of } v \text { for every } L \in \mathscr{L}_{\bar{\gamma}(U)},
$$

or all lines of $\bar{\gamma}(U)$ are variable on $H$ relative to $\gamma$, i.e.

$$
k_{L}^{\gamma}(u, v, q) \text { is dependent on } v \text { for every } L \in \mathscr{L}_{\bar{\gamma}(U)} .
$$

$\bar{\gamma}(U)$ is said to be constant or variable on $H$, respectively.

Lemma 5.1 [5]. Let $\Gamma$ be a 1PI diagram, $H \in \mathscr{H}$ and $\mathscr{W}_{c}^{H}$ the set of all $\Gamma$-forests which are complete on $H$. Then

$$
\hat{R}_{\Gamma}(k, q ; s, \mu, a)=\sum_{U \in \mathscr{W}_{c}^{H}} \hat{X}_{U}(k, q ; s, \mu, a),
$$


where

$$
\hat{X}_{U}(k, q ; s, \mu, a)=\left.\left(1-\hat{\tau}_{\Gamma}\right) \hat{Y}_{\Gamma}\left(k^{\Gamma}, q^{\Gamma} ; s^{\Gamma}, \mu, a\right)\right|_{k^{\Gamma}=k ; q^{\Gamma}=q ; s^{\Gamma}=s},
$$

and

$$
\hat{Y}_{\gamma}\left(k^{\gamma}, q^{\gamma} ; s^{\gamma}, \mu, a\right)=\hat{I}_{\bar{\gamma}(U)}\left(k^{\gamma}, q^{\gamma} ; s^{\gamma}, \mu, a\right) \cdot S_{\gamma} \prod_{i=1}^{c} \hat{f}\left(\gamma_{i}\right) \hat{Y}_{\gamma_{i}}\left(k^{\gamma_{i}}, q^{\gamma_{i}} ; s^{\gamma_{i}}, \mu, a\right),
$$

for any $\gamma \in U, \gamma_{1}, \ldots, \gamma_{c}$ being the maximal elements of $U(\gamma)$. For minimal $\gamma$ set $\hat{Y}_{\gamma}=\hat{I}_{\gamma}$. $\hat{f}(\gamma)$ is defined by

$$
\hat{f}(\gamma)=\left\{\begin{array}{lll}
1-\hat{\tau}_{\gamma} & \text { if } & \gamma \in \mathscr{B}(U) \\
-\hat{\tau}_{\gamma} & \text { if } & \gamma \notin \mathscr{B}(U),
\end{array}\right.
$$

where $\mathscr{B}(U)$ is the set of all $\gamma \in U$ having $\bar{\gamma}(U)$ variable on $H$ and being a maximal element of $U(\tau)$ for some $\tau \in U$ having $\bar{\tau}(U)$ constant on $H$.

Let $U$ be a $\Gamma$-forest, $\gamma \in U$ and $\gamma_{1}, \ldots, \gamma_{c}$ the maximal elements of $U(\gamma)$. Then $S_{\gamma}$ means a linear substitution

$$
S_{\gamma}: k^{\gamma_{2}} \rightarrow k^{\gamma_{1}}\left(k^{\gamma}\right), \quad q^{\gamma_{2}} \rightarrow q^{\gamma_{2}}\left(k^{\gamma}, q^{\gamma}\right), \quad s^{\gamma_{2}} \rightarrow s^{\gamma},
$$

where the $k^{\gamma}$-dependence of $q^{\gamma_{z}}$ is only by the explicit $k^{\gamma}$-dependence of external lines of $\gamma_{i}$, and $k^{\gamma_{2}}$ is independent of $q^{\gamma}$. Especially, if $H \in \mathscr{H}$ is given by variable $(v)$ and constant $(u)$ and if $\bar{\gamma}(U)$ is constant, then $q^{\gamma_{2}}\left(k^{\gamma}, q^{\gamma}\right)$ depends only on $u$ and $q^{\gamma}$.

The prove of the UV-convergence conditions (I) is along the lines of the proof [4]. Let $H \in \mathscr{H}$ be defined by variable $(v)=\left(v_{1}, \ldots, v_{d}\right)$ and constant $(u)=\left(u_{1}, \ldots, u_{r}\right)$, and let $U \in \mathscr{W}_{c}^{H}$. For $\gamma \in U$ define

$$
M_{U}(\gamma)=4 \sum_{\tau} m(\bar{\tau}(U))
$$

where the sum is over all $\tau \in U(\gamma) \cup\{\gamma\}, \bar{\tau}(U)$ variable, and $m(\bar{\tau}(U))$ is the number of loops in $\bar{\tau}(U)$. For $\gamma=\Gamma, M_{U}(\Gamma) \geqq 4 d[4]$. Then the following lemma holds.

Lemma 5.2. For every $\gamma \in U$,

$$
\text { 1. } \overline{\operatorname{degr}}_{\hat{v}} \hat{Y}_{\gamma}\left(k^{\gamma}(u, v, q), q^{\gamma} ; s^{\gamma}, \mu, a\right) \leqq-M_{U}(\gamma) \text { for } \bar{\gamma}(U) \text { constant }
$$

equality holding only if $M_{U}(\gamma)=0$.

2. $\overline{\operatorname{degr}}_{\widehat{v q}^{\gamma} s^{\gamma}} \hat{Y}_{\gamma}\left(k^{\gamma}(u, v, q), q^{\gamma} ; s^{\gamma}, \mu, a\right) \leqq \delta(\gamma)-M_{U}(\gamma)$ for $\bar{\gamma}(U)$ variable.

For $\Gamma$,

$$
\text { 3. } \overline{\operatorname{degr}}_{\hat{v}} \hat{R}_{\Gamma}(k(u, v, q), q ; s, \mu, a)<-4 d \text {. }
$$

The proof of Lemma 5.2 is quite similar as the proof of [4, Lemma 5.2], the only difference being the appearence of the mass parameters $s^{\gamma}$. Nevertheless, as Lemma 5.2 of [4] is a consequence of the general properties of a subtraction operator listed in Lemma 3.1 of [4], the validity of the above lemma is based on the corresponding conditions of generalized subtraction operators listed in Lemma 4.2. For this reason, the proof is left as an exercise to the reader. By Lemma 5.2, the UV-conditions (I) are satisfied. 


\section{Proof of the IR-Convergence Conditions (II)}

To prove the inequalities (II) we will use the technical notion of an augmented diagram [10] $\tilde{\Gamma}$ of $\Gamma$. $\tilde{\Gamma}$ is constructed by collecting all external lines of $\Gamma$ into a new vertex $B_{0}$. Momentum conservation in $\Gamma$ implies momentum conservation in $B_{0}$. If $\Gamma$ has more than one external line, $\widetilde{\Gamma}$ is $1 \mathrm{PI}$ if $\Gamma$ is. If $\Gamma$ has no external lines, then $\tilde{\Gamma}=\Gamma$.

More precisely, let

$$
\Gamma=\left(\mathscr{L}_{\Gamma}, \mathscr{E}_{\Gamma}, \mathscr{B}_{\Gamma}, \phi_{\Gamma}, \psi_{\Gamma}\right)
$$

be a 1 PI diagram having at least one external line. Then the augmented diagram $\tilde{\Gamma}$ of $\Gamma$ is defined by

$$
\tilde{\Gamma}=\left(\mathscr{L}_{\tilde{\Gamma}}, \mathscr{E}_{\tilde{\Gamma}}, \mathscr{B}_{\tilde{\Gamma}}, \phi_{\tilde{\Gamma}}, \psi_{\tilde{\Gamma}}\right),
$$

where

$$
\begin{aligned}
\mathscr{L}_{\tilde{\Gamma}} & =\mathscr{L}_{\Gamma} \cup \mathscr{E}_{\Gamma}\left(\mathscr{L}_{\Gamma} \cap \mathscr{E}_{\Gamma}=\emptyset\right), \\
\mathscr{E}_{\tilde{\Gamma}} & =\emptyset, \\
\mathscr{B}_{\tilde{\Gamma}} & =\mathscr{B}_{\Gamma} \cup\left\{B_{0}\right\}, \quad B_{0} \notin \mathscr{B}_{\Gamma},
\end{aligned}
$$

and

$$
\begin{aligned}
& \phi_{\tilde{\Gamma}}: \mathscr{L}_{\tilde{\Gamma}} \rightarrow \mathscr{B}_{\tilde{\Gamma}} \times \mathscr{B}_{\tilde{\Gamma}}, \\
& \phi_{\tilde{\Gamma}}(L)=\phi_{\Gamma}(L) \quad \text { if } \quad L \in \mathscr{L}_{\Gamma} \text {, } \\
& \phi_{\tilde{\Gamma}}(E)=\left(B_{0}, \psi_{\Gamma}(E)\right) \quad \text { if } \quad E \in \mathscr{E}_{\Gamma} .
\end{aligned}
$$

The domain of $\psi_{\tilde{\Gamma}}: \mathscr{E}_{\tilde{\Gamma}} \rightarrow \mathscr{B}_{\tilde{\Gamma}}$ is empty. Every line $L \in \mathscr{L}_{\tilde{\Gamma}} \backslash \mathscr{L}_{\Gamma}$ is called a $q$-Linie of $\tilde{\Gamma}$.

We now state two lemmas which are consequences of the assumed nonexceptionality of the external momenta and the IR-constraints (3.9). They will be useful later for the induction through a complete forest.

Let $\Gamma$ be a $1 \mathrm{PI}$ diagram having $m$ loops and $\tilde{\Gamma}$ the augmented diagram of $\Gamma$. For every 1PI subdiagram $\gamma$ of $\Gamma$, the IR-subtraction degree is given by

$$
\varrho(\gamma)=4+\sum_{B \in \mathscr{B}_{\gamma}}[\varrho(B)-4]-\sum_{k} e_{k}(\gamma) r_{k},
$$

where the $\varrho(B)$ are constrained by (3.9). Let $E(\Gamma)$ be the number of $q$-lines of $\widetilde{\Gamma}$ and $\gamma_{1}, \ldots, \gamma_{c}$ be mutually disjoint 1PI subdiagrams of $\Gamma, \Gamma \notin\left\{\gamma_{1}, \ldots, \gamma_{c}\right\}$. form

Suppose that an arbitrary parametrization of the loop momenta of $\Gamma$ of the

$$
k_{i}(w, p)=\sum_{j=1}^{m} D_{i j} w_{j}+p_{i}, \quad i=1, \ldots, m,
$$

is given, where $\operatorname{det}(D) \neq 0$ and $p_{i}$ are fixed momenta, so that for all line momenta $l_{L}$, $l \in \mathscr{L}_{\Gamma}$,

$$
l_{L}=l_{L}(w, q, p)=\sum_{j=1}^{m}\left(C_{L}\right)_{j} w_{j}+Q_{L}(q)+P_{L}(p),
$$

where $Q_{L}, P_{L}$ are linear. 
Let $\bar{\Lambda}_{0}, \bar{\Lambda}_{1}, \ldots, \bar{\Lambda}_{b}$ be the mutually disjoint, connected subdiagrams of $\tilde{\Gamma} / \gamma_{1} \ldots \gamma_{c}$, which are spanned by the $q$-lines and the lines $L \in \mathscr{L}_{\Gamma}$ having $Q_{L}(q)+P_{L}(p) \neq 0$, and so that for $E(\Gamma) \geqq 1$ always $B_{0} \in \bar{\Lambda}_{0}$ (cp. the definition of $\widetilde{\Gamma}$ ) and for $E(\Gamma)=0: \bar{\Lambda}_{0}=\emptyset$. By momentum conservation, all $\bar{\Lambda}_{1}, \ldots, \bar{\Lambda}_{b}$ are $1 \mathrm{PI}$, hence their number of loops $m\left(\bar{\Lambda}_{i}\right) \geqq 1, i=1, \ldots, b$. Furthermore, let $\lambda_{1}, \ldots, \lambda_{e}$ be the elements of $\left\{\gamma_{1}, \ldots, \gamma_{c}\right\}$ which corresponds in $\tilde{\Gamma} / \gamma_{1} \ldots \gamma_{c}$ to reduced vertices not contained in $\mathscr{B}_{\Lambda_{0}} \cup \ldots \cup \mathscr{B}_{\Lambda_{b}}$.

Lemma 6.1. If the external momenta of $\Gamma$ are non-exceptional, we have

$$
m\left(\bar{\Lambda}_{0}\right) \geqq E(\Gamma)-1 .
$$

If in addition for all internal vertices $B \in \mathscr{B}_{\Gamma} r(B) \geqq 4$ and $\varrho(B) \geqq 4$, the inequality

$$
r(T)+\sum_{i=1}^{e} \max \left(0, \varrho\left(\lambda_{i}\right)\right)-4 m(T)>-4 m\left(\Gamma / \gamma_{1} \ldots \gamma_{c}\right)
$$

holds, where $T=\left(\widetilde{\Gamma} / \gamma_{1} \ldots \gamma_{c}\right) / \bar{\Lambda}_{0} \ldots \bar{\Lambda}_{b}$, and $r(T)$ and $m(T)$ are the IR-divergence degree of $T$ and the number of loops in $T$, respectively.

For non-exceptional external momenta and $E(\Gamma) \geqq 2, \bar{\Lambda}_{0}$ is $1 \mathrm{PI}$. If $E(\Gamma)=0$, $\bar{\Lambda}_{0}=\emptyset$, and for $E(\Gamma)=1, \mathscr{L}_{\bar{\Lambda}_{0}}$ consists of one $q$-Linie.

Proof. We always have $m\left(\bar{\Lambda}_{0}\right) \geqq 0$. If $E(\Gamma) \geqq 2$, and the external momenta of $\Gamma$ are non-exceptional, the diagram spanned by the lines of $\mathscr{L}_{\bar{\Lambda}_{0}} \cap \mathscr{L}_{\Gamma / \gamma_{1} \ldots \gamma_{c}}$ is connected and contains all external vertices of $\Gamma / \gamma_{1} \ldots \gamma_{c}$. This proves the first statement.

To prove the second statement, we first note that if $\bar{\Lambda}_{0}=\tilde{\Gamma} / \gamma_{1} \ldots \gamma_{c},(6.5)$ is trivial. Thus, let us assume that $\bar{\Lambda}_{0} \neq \widetilde{\Gamma} / \gamma_{1} \ldots \gamma_{c}$. The number of loops in $T$ satisfies

$$
\begin{aligned}
m(T) & =m\left(\tilde{\Gamma} / \gamma_{1} \ldots \gamma_{c}\right)-\sum_{i=0}^{b} m\left(\bar{\Lambda}_{i}\right) \\
& \leqq m\left(\tilde{\Gamma} / \gamma_{1} \ldots \gamma_{c}\right)-(E(\Gamma)-1)\left(1-\delta_{E(\Gamma), 0}\right)-\sum_{i=1}^{b} m\left(\bar{\Lambda}_{i}\right) \\
& =m\left(\Gamma / \gamma_{1} \ldots \gamma_{c}\right)-\sum_{i=1}^{b} m\left(\bar{\Lambda}_{i}\right) .
\end{aligned}
$$

Furthermore

$$
\begin{aligned}
r(T)-4 m(T) \geqq & 4+\sum_{B \in \mathscr{B}_{T}}[r(B)-4]-4 m(T) \quad(\text { by }(2.30), T \text { has no external legs }) \\
\geqq & +\sum_{B \in \mathscr{B}_{T} \cap \mathscr{B}_{T^{\prime}}}[r(B)-4]+\sum_{i=1}^{e}\left(\sum_{k} e_{k}\left(\lambda_{i}\right) r_{k}-4\right) \\
& +\sum_{i=1}^{b}\left(\sum_{k} e_{k}\left(\bar{\Lambda}_{i}\right) r_{k}-4\right)+\left(\sum_{k} e_{k}\left(\bar{\Lambda}_{0}\right) r_{k}-4\right)\left(1-\delta_{E(\Gamma), 0}\right) \\
& -4 m\left(\Gamma / \gamma_{1} \ldots \gamma_{c}\right)+4 \sum_{i=1}^{b} m\left(\bar{\Lambda}_{i}\right) \quad[\mathrm{by}(2.31)] \\
& >\sum_{i=1}^{e}\left(\sum_{k} e_{k}\left(\lambda_{i}\right) r_{k}-4\right)-4 m\left(\Gamma / \gamma_{1} \ldots \gamma_{c}\right)
\end{aligned}
$$


where we have used $r_{k}>0, m\left(\bar{\Lambda}_{i}\right) \geqq 1$ for all $i=1, \ldots, b$ and that $r(B) \geqq 4$ for all internal vertices $B \in \mathscr{B}_{\Gamma}\left(\mathscr{B}_{T} \cap \mathscr{B}_{\Gamma}\right.$ contains only internal vertices of $\left.\Gamma\right)$. Finally

$$
\begin{aligned}
r(T) & +\sum_{i=1}^{e} \max \left(0, \varrho\left(\lambda_{i}\right)\right)-4 m(T) \\
& >\sum_{i=1}^{e}\left(\sum_{k} e_{k}\left(\lambda_{i}\right) r_{k}-4\right)+\sum_{i=1}^{e} \max \left(0,4-\sum_{k} e_{k}\left(\lambda_{i}\right) r_{k}+\sum_{B \in \mathscr{B}_{\lambda_{i}}}[\varrho(B)-4]\right) \\
& -4 m\left(\Gamma / \gamma_{1} \ldots \gamma_{c}\right) \geqq-4 m\left(\Gamma / \gamma_{1} \ldots \gamma_{c}\right),
\end{aligned}
$$

where we have used that all $B \in \mathscr{B}_{\lambda_{i}}$ are internal vertices and for them $\varrho(B) \geqq 4$.

To state the second lemma, let $\bar{\Sigma}_{1}, \ldots, \bar{\Sigma}_{b}$ be the connected, mutually disjoint subdiagrams of $\Gamma / \gamma_{1} \ldots \gamma_{c}$ spanned by the lines $L \in \mathscr{L}_{\Gamma / \gamma_{1} \ldots \gamma_{c}}$ satisfying $P_{L}(p) \neq 0$. Every $\bar{\Sigma}_{i}$ is $1 \mathrm{PI}$ and satisfies $m\left(\bar{\Sigma}_{i}\right) \geqq 1$.

Lemma 6.2. Set $T=\left(\Gamma / \gamma_{1} \ldots \gamma_{c}\right) / \bar{\Sigma}_{1} \ldots \bar{\Sigma}_{b}$. If all vertices $B \in \mathscr{B}_{\Gamma}$ satisfy $\varrho(B) \leqq 4$ and $\varrho(B) \leqq r(B)$, the inequality

$$
r(T)+\sum_{i=1}^{c} \max \left(0, \varrho\left(\gamma_{i}\right)\right)-4 m(T) \geqq \varrho(\Gamma)-4 m\left(\Gamma / \gamma_{1} \ldots \gamma_{c}\right)
$$

holds.

Proof. Let $\lambda_{1}, \ldots, \lambda_{e}$ be the elements of $\left\{\gamma_{1}, \ldots, \gamma_{c}\right\}$ which corresponds in $\Gamma / \gamma_{1} \ldots \gamma_{c}$ to those vertices not contained in $\mathscr{B}_{\bar{\Sigma}_{1}} \cup \ldots \cup \mathscr{B}_{\bar{\Sigma}_{b}}$. Then, using $m(T)=m\left(\Gamma / \gamma_{1} \ldots \gamma_{c}\right)$ $-\sum_{i=1}^{b} m\left(\bar{\Sigma}_{i}\right)$, we get

$$
\begin{aligned}
& r(T)+\sum_{i=1}^{c} \max \left(0, \varrho\left(\gamma_{i}\right)\right)-4 m(T) \\
&= {\left[4+\sum_{B \in \mathscr{B}_{T \cap \mathscr{B}_{\Gamma}}}[r(B)-4]+\sum_{i=1}^{e}\left(\sum_{k} e_{k}\left(\lambda_{i}\right) r_{k}-4\right)+\sum_{i=1}^{b}\left(\sum_{k} e_{k}\left(\bar{\Sigma}_{i}\right) r_{k}-4\right)\right.} \\
&\left.-\sum_{k} e_{k}(T) r_{k}\right]-\left[4 m\left(\Gamma / \gamma_{1} \ldots \gamma_{c}\right)-4 \sum_{i=1}^{b} m\left(\bar{\Sigma}_{i}\right)\right]+\sum_{i=1}^{c} \max \left(0, \varrho\left(\gamma_{i}\right)\right) \\
& \geqq+\sum_{B \in \mathscr{B}_{T} \cap \mathscr{B}_{\Gamma}}[r(B)-4]+\sum_{i=1}^{e}\left(\sum_{k} e_{k}\left(\lambda_{i}\right) r_{k}-4\right)-\sum_{k} e_{k}(T) r_{k} \\
&+\sum_{i=1}^{c} \max \left(0,4-\sum_{k} e_{k}\left(\gamma_{i}\right) r_{k}+\sum_{B \in \mathscr{B}_{\gamma_{i}}}(\varrho(B)-4)\right)-4 m\left(\Gamma / \gamma_{1} \ldots \gamma_{c}\right)
\end{aligned}
$$

(by $r_{k}>0$ and $m\left(\bar{\Sigma}_{i}\right) \geqq 1$ )

$$
\geqq\left(4+\sum_{B \in \mathscr{B}_{\Gamma}}[\varrho(B)-4]-\sum_{k} e_{k}(\Gamma) r_{k}\right)-4 m\left(\Gamma / \gamma_{1} \ldots \gamma_{c}\right),
$$

where we have used $e_{k}(T)=e_{k}(\Gamma)$ for all $k$ and $\varrho(B) \leqq r(B), \varrho(B) \leqq 4$ for all vertices $B \in \mathscr{B}_{\Gamma}$.

Using the mechanism of complete forests we now prove the IR-power counting conditions (II). The starting point is Lemma 5.1. The idea of proof is along the lines of [10]. 
Always in the following let $\Gamma$ be a 1PI Feynman diagram and $m$ the number of loops in $\Gamma, H \in \mathscr{H}$ given by variable $(v)=\left(v_{1}, \ldots, v_{d}\right)$ and constant $(u)=\left(u_{1}, \ldots, u_{r}\right)$, and let $U$ be a $\Gamma$-forest which is complete on $H$. At first, for every $\gamma \in U$ we define

$$
N_{U}(\gamma)=4 \sum_{\tau} m(\bar{\tau}(U)) \text {. }
$$

The sum is over all $\tau \in U(\gamma) \cup\{\gamma\}, \bar{\tau}(U)$ constant, and $m(\bar{\tau}(U))$ is the number of loops in $\bar{\tau}(U)$. For $\gamma=\Gamma, N_{U}(\Gamma) \leqq 4 r$. This follows from $M_{U}(\Gamma)=4 m-N_{U}(\Gamma) \geqq 4 d$ and $r+d=m($ cf. $(5.10))$.

The next lemma states the action of the operators $\hat{\tau}_{\gamma}$ onto $\hat{Y}_{\gamma}$.

Lemma 6.3. For every $\gamma \in U$, the following inequalities hold.

1. $\underline{\operatorname{degr}}_{\hat{u} \mid q^{\gamma} v\left(s^{\gamma}-1\right)} \hat{\tau}_{\gamma} \hat{Y}_{\gamma}\left(k^{\gamma}(u, v, q), q^{\gamma} ; s^{\gamma}, \mu, a\right)$

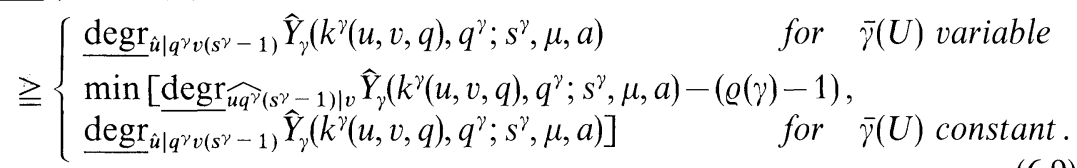

2. $\underline{\operatorname{degr}}_{u q^{\gamma}\left(s^{\gamma}-1\right) \mid v} \hat{\tau}_{\gamma} \hat{Y}_{\gamma}\left(k^{\gamma}(u, v, q), q^{\gamma} ; s^{\gamma}, \mu, a\right)$

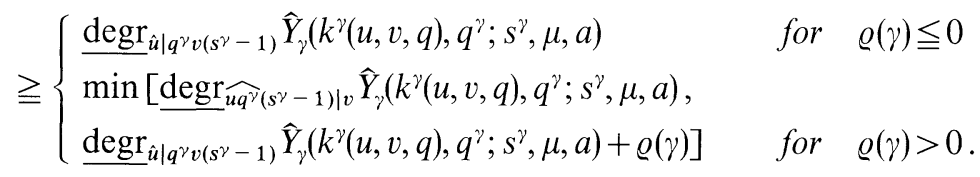

3. Suppose $\bar{\gamma}(U)$ is variable. Then the inequality

$$
\begin{aligned}
& \operatorname{degr}_{\widehat{u q^{\gamma}}\left(s^{\gamma}-1\right) \mid v}\left(1-\hat{\tau}_{\gamma}\right) \hat{Y}_{\gamma}\left(k^{\gamma}(u, v, q), q^{\gamma} ; s^{\gamma}, \mu, a\right) \\
& \quad \geqq \underline{\operatorname{degr}}_{\hat{u} \mid q^{\gamma} v\left(s^{\gamma}-1\right)} \hat{Y}_{\gamma}\left(k^{\gamma}(u, v, q), q^{\gamma} ; s^{\gamma}, \mu, a\right)+\max (0, \varrho(\gamma))
\end{aligned}
$$

holds.

Proof. $\hat{\tau}_{\gamma}$ is written in the form

$$
\hat{\tau}_{\gamma}=\hat{\tau}_{\gamma 1}+\hat{\tau}_{\gamma 2}-\hat{\tau}_{\gamma 1} \hat{\tau}_{\gamma 2}=\hat{\tau}_{\gamma 1}+\left(1-\hat{\tau}_{\gamma 1}\right) \hat{\tau}_{\gamma 2}
$$

where

$$
\hat{\tau}_{\gamma 1}=\hat{t}_{q^{\gamma}\left(s^{\gamma}-1\right)}^{\varrho(\gamma)-1}, \quad \hat{\tau}_{\gamma 2}=\hat{t}_{q^{\gamma} s^{\gamma}}^{\partial(\gamma)} .
$$

If $\bar{\gamma}(U)$ is variable, every factor in the denominator of $\hat{Y}_{\gamma}$ depends on $v$ or is independent of $q^{\gamma}$ and $\left(s^{\gamma}-1\right)$.

1.a) Using (2.11), Lemma 4.4.3 and Lemma 4.3, we get

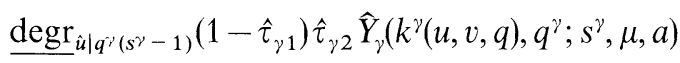

$$
\begin{aligned}
& \geqq \underline{\operatorname{degr}} \hat{u}_{\hat{u} \mid q^{\gamma} v\left(s^{\gamma}-1\right)} \hat{\tau}_{\gamma 2} \hat{Y}_{\gamma}\left(k^{\gamma}(u, v, q), q^{\gamma} ; s^{\gamma}, \mu, a\right) \\
& \geqq \underline{\operatorname{degr}}_{\hat{u} \mid q^{\gamma} v\left(s^{\gamma}-1\right)} \hat{Y}_{\gamma}\left(k^{\gamma}(u, v, q), q^{\gamma} ; s^{\gamma}, \mu, a\right) \text {. }
\end{aligned}
$$

b) Using (5.7b), (5.8) and Lemma 4.4, we get

$$
\begin{aligned}
& \underline{\operatorname{degr}}_{\hat{u} \mid q^{\gamma} v\left(s^{\gamma}-1\right)} \hat{\tau}_{\gamma 1} \hat{Y}_{\gamma}\left(k^{\gamma}(u, v, q), q^{\gamma} ; s^{\gamma}, \mu, a\right) \\
& \geqq \begin{cases}\frac{\operatorname{degr}}{\hat{u} \mid q^{\gamma} v\left(s^{\gamma}-1\right)} \hat{Y}_{\gamma}\left(k^{\gamma}(u, v, q), q^{\gamma} ; s^{\gamma}, \mu, a\right) & \text { for } \quad \bar{\gamma}(U) \text { variable } \\
\underline{\operatorname{degr}} \hat{u q^{\gamma}\left(s^{\gamma}-1\right) \mid v} \hat{Y}_{\gamma}\left(k^{\gamma}(u, v, q), q^{\gamma} ; s^{\gamma}, \mu, a\right)-(\varrho(\gamma)-1) & \text { for } \quad \bar{\gamma}(U) \text { constant. }\end{cases}
\end{aligned}
$$


Taking the minimum, assertion 1. follows.

2.a) $\operatorname{degr}_{\widehat{u q^{\gamma}}\left(s^{\gamma}-1\right) \mid v}\left(1-\hat{\tau}_{\gamma 1}\right) \hat{\tau}_{\gamma 2} \hat{Y}_{\gamma}\left(k^{\gamma}(u, v, q), q^{\gamma} ; s^{\gamma}, \mu, a\right)$

$\geqq \underline{\operatorname{degr}_{\hat{u} \mid q^{\gamma} v\left(s^{\gamma}-1\right)}} \hat{\tau}_{\gamma_{2}} \hat{Y}_{\gamma}\left(k^{\gamma}(u, v, q), q^{\gamma} ; s^{\gamma}, \mu, a\right)+\max (0, \varrho(\gamma))$,

(by Lemma 4.4.4)

$\geqq \underline{\operatorname{degr}_{\hat{u} \mid q^{\gamma} v\left(s^{\gamma}-1\right)}} \hat{Y}_{\gamma}\left(k^{\gamma}(u, v, q), q^{\gamma} ; s^{\gamma}, \mu, a\right)+\max (0, \varrho(\gamma))$,

(by Lemma 4.3).

b) Note that $\varrho(\gamma) \leqq 0$ implies $\hat{\tau}_{\gamma 1} \hat{Y}_{\gamma}=0$. If $\varrho(\gamma)>0$, we get

$$
\begin{aligned}
& \frac{\operatorname{degr} \widehat{r}_{\widehat{u \gamma^{\gamma}}\left(s^{\gamma}-1\right) \mid v} \hat{\tau}_{\gamma 1} \hat{Y}_{\gamma}\left(k^{\gamma}(u, v, q), q^{\gamma} ; s^{\gamma}, \mu, a\right)}{} \geqq \underline{\operatorname{degr}_{\widehat{u q^{\gamma}}\left(s^{\gamma}-1\right) \mid v}} \hat{Y}_{\gamma}\left(k^{\gamma}(u, v, q), q^{\gamma} ; s^{\gamma}, \mu, a\right) \quad \text { (by Lemma 4.4.1). }
\end{aligned}
$$

Assertion 2. now follows by taking the minimum.

3. Let $\bar{\gamma}(U)$ be variable. Then Lemma 4.4 .4 yields

$$
\begin{aligned}
& \underline{\operatorname{degr}}_{\widehat{u q^{\gamma}}\left(s^{\gamma}-1\right) \mid v}\left(1-\hat{\tau}_{\gamma 1}\right) \hat{Y}_{\gamma}\left(k^{\gamma}(u, v, q), q^{\gamma} ; s^{\gamma}, \mu, a\right) \\
& \quad \geqq \underline{\operatorname{degr}}_{\hat{u} \mid q^{\gamma} v\left(s^{\gamma}-1\right)} \hat{Y}_{\gamma}\left(k^{\gamma}(u, v, q), q^{\gamma} ; s^{\gamma}, \mu, a\right)+\max (0, \varrho(\gamma)) .
\end{aligned}
$$

Using $1-\hat{\tau}_{\gamma}=\left(1-\hat{\tau}_{\gamma 1}\right)\left(1-\hat{\tau}_{\gamma_{2}}\right)$ and 2.a, the assertion follows.

Using Lemma 6.3, we get the following lemma which states lower bounds on the IR-degrees of the functions defined in Lemma 5.1.

Lemma 6.4. 1. For every $\gamma \in U$

$$
\begin{gathered}
\text { degr }_{\widehat{u q^{\gamma}}\left(s^{\gamma}-1\right) \mid v} \hat{Y}_{\gamma}\left(k^{\gamma}(u, v, q), q^{\gamma} ; s^{\gamma}, \mu, a\right) \geqq \varrho(\gamma)-N_{U}(\gamma) \quad \text { if } \quad \bar{\gamma}(U) \text { constant } . \\
\frac{\operatorname{degr}_{\hat{u} \mid q^{\gamma} v\left(s^{\gamma}-1\right)} \hat{Y}_{\gamma}\left(k^{\gamma}(u, v, q), q^{\gamma} ; s^{\gamma}, \mu, a\right) \geqq-N_{U}(\gamma)}{\left(=\text { holding only if } N_{U}(\gamma)=0\right) .}
\end{gathered}
$$

2. Let $\gamma \in U$ and $\lambda$ be a maximal element of $U(\gamma)$.

a)

$$
\begin{gathered}
\underline{\operatorname{degr}}_{\hat{u} \mid q^{\gamma} v\left(s^{\gamma}-1\right)} S_{\gamma} \hat{\tau}_{\lambda} \hat{Y}_{\lambda}\left(k^{\lambda}(u, v, q), q^{\lambda} ; s^{\lambda}, \mu, a\right) \geqq-N_{U}(\lambda) \\
\left(=\text { holding only if } N_{U}(\lambda)=0\right) .
\end{gathered}
$$

In particular

$$
\underline{\operatorname{degr}}_{\hat{u} \mid q^{\Gamma} v\left(s^{\Gamma}-1\right)} \hat{\tau}_{\Gamma} \hat{Y}_{\Gamma}\left(k^{\Gamma}(u, v, q), q^{\Gamma} ; s^{T}, \mu, a\right)>-4 r .
$$

b)

$$
\begin{gathered}
\operatorname{degr}_{\hat{u} \mid q^{\gamma} v\left(s^{\gamma}-1\right)} S_{\gamma}\left(1-\hat{\tau}_{\lambda}\right) \hat{Y}_{\lambda}\left(k^{\lambda}(u, v, q), q^{\lambda} ; s^{\lambda}, \mu, a\right) \geqq-N_{U}(\lambda) \\
\left(=\text { holding only if } N_{U}(\lambda)=0\right),
\end{gathered}
$$

for $\bar{\gamma}(U)$ constant and $\bar{\lambda}(U)$ variable.

c) $\quad \operatorname{degr}_{\widehat{u q^{\gamma}}\left(s^{\gamma}-1\right) \mid v} S_{\gamma} \hat{\tau}_{\lambda} \hat{Y}_{\lambda}\left(k^{\lambda}(u, v, q), q^{\lambda} ; s^{\lambda}, \mu, a\right) \geqq \max (0, \varrho(\lambda))-N_{U}(\lambda)$

for $\bar{\gamma}(U)$ constant and $\bar{\lambda}(U)$ constant.

d) $\underline{\operatorname{degr}}_{\widehat{u q^{\gamma}}\left(s^{\gamma}-1\right) \mid v} S_{\gamma}\left(1-\hat{\tau}_{\lambda}\right) \hat{Y}_{\lambda}\left(k^{\lambda}(u, v, q), q^{\lambda} ; s^{\lambda}, \mu, a\right) \geqq \max (0, \varrho(\lambda))-N_{U}(\lambda)$

for $\bar{\gamma}(U)$ constant and $\bar{\lambda}(U)$ variable.

The statements a)-d) are also valid without $S_{\gamma}$ and with $q^{\lambda},\left(s^{\lambda}-1\right)$ instead of $q^{\gamma}$, $\left(s^{\gamma}-1\right)$. 
Proof. By complete induction.

1. For minimal $\gamma$, we have $\gamma=\bar{\gamma}(U)$ and $\hat{Y}_{\gamma}=\hat{I}_{\gamma}$. In general

$$
\underline{\operatorname{degr}}_{\hat{u} \mid q^{\gamma} v\left(s^{\gamma}-1\right)} \hat{I}_{\gamma}\left(k^{\gamma}(u, v, q), q^{\gamma} ; s^{\gamma}, \mu, a\right) \geqq 0,
$$

because of (2.11) for every line $L \in \mathscr{L}_{\gamma}$.

If $\bar{\gamma}(U)$ is constant, let $\Sigma_{1}, \ldots, \Sigma_{b}$ be all 1 PI subdiagrams of $\gamma$ which are spanned by the lines $L \in \mathscr{L}_{\gamma}$ satisfying $k_{L}^{\gamma}(0,0, q) \neq 0$. Because of $\varrho(B) \leqq 4$ and $\varrho(B) \leqq r(B)$ for all $B \in \mathscr{B}_{\gamma}$, using Lemma 6.2 and writing $T=\gamma / \Sigma_{1} \ldots \Sigma_{b}$, we get

$$
\begin{aligned}
& \operatorname{degr}_{\widehat{u q^{\gamma}}\left(s^{\gamma}-1\right) \mid v} \hat{I}_{\gamma}\left(k^{\gamma}(u, v, q), q^{\gamma} ; s^{\gamma}, \mu, a\right) \\
& \geqq \operatorname{degr}_{\widehat{u q^{\gamma}}\left(s^{\gamma}-1\right)} \hat{I}_{T}\left(k^{\gamma}(u, v=0, q=0), q^{\gamma} ; s^{\gamma}, \mu, a\right) \\
& =r(T)-4 m(T) \geqq \varrho(\gamma)-4 m(\gamma)=\varrho(\gamma)-N_{U}(\gamma) \text {. }
\end{aligned}
$$

This proves the first statement of the lemma for minimal $\gamma \in U$.

2. Let $\gamma \in U$ and $\lambda$ be a maximal element of $U(\gamma)$. By hypothesis of induction, $\hat{Y}_{\lambda}$ satisfies Lemma 6.4.1.

To prove the statements $2 \mathrm{a}$ ) and $2 \mathrm{~b}$ ) of the lemma, we use Lemma 6.3.1 and get

$$
\begin{gathered}
\operatorname{degr}_{\hat{u} \mid q^{\lambda} v\left(s^{\lambda}-1\right)} \hat{\tau}_{\lambda} \hat{Y}_{\lambda}\left(k^{\lambda}(u, v, q), q^{\lambda} ; s^{\lambda}, \mu, a\right) \geqq-N_{U}(\lambda) \\
\left(=\text { holding only if } N_{U}(\lambda)=0\right)
\end{gathered}
$$

and

$$
\begin{aligned}
& \underline{\operatorname{degr}}_{\hat{u} \mid q^{\lambda} v\left(s^{\lambda}-1\right)}\left(1-\hat{\tau}_{\lambda}\right) \hat{Y}_{\lambda}\left(k^{\lambda}(u, v, q), q^{\lambda} ; s^{\lambda}, \mu, a\right) \\
& \quad \geqq \min \left[\underline{\operatorname{degr}}_{\hat{u} \mid q^{\lambda} v\left(s^{\lambda}-1\right)} \hat{Y}_{\lambda}, \underline{\operatorname{degr}_{\hat{u} \mid q^{\lambda} v\left(s^{\lambda}-1\right)}} \hat{\tau}_{\lambda} \hat{Y}_{\lambda}\right] \\
& \quad \geqq-N_{U}(\lambda) \\
& \quad\left(=\text { holding only if } N_{U}(\lambda)=0\right) .
\end{aligned}
$$

$S_{\gamma}$ is a linear transformation

$$
S_{\gamma}: q^{\lambda} \rightarrow q^{\lambda}\left(k^{\gamma}(u, v, q), q^{\gamma}\right), \quad s^{\lambda} \rightarrow s^{\gamma},
$$

where the $k^{\gamma}$-dependence of $q^{\lambda}$ is only by the explicit $k^{\gamma}$-dependence of the external lines of $\lambda$, i.e. lines which belong to $\mathscr{L}_{\bar{\gamma}(U)}$. The denominator of $\hat{\tau}_{\lambda} \hat{Y}_{\lambda}$ is independent of $q^{\lambda}$ and $\left(s^{\lambda}-1\right)$, hence

$$
\begin{aligned}
& \frac{\operatorname{degr}_{\hat{u} \mid q^{\gamma} v\left(s^{\gamma}-1\right)} S_{\gamma} \hat{\tau}_{\lambda} \hat{Y}_{\lambda}\left(k^{\lambda}(u, v, q), q^{\lambda} ; s^{\lambda}, \mu, a\right)}{\quad \geqq \operatorname{degr}_{\hat{u} \mid q^{\lambda} v\left(s^{\lambda}-1\right)} \hat{\tau}_{\lambda} \hat{Y}_{\lambda}\left(k^{\lambda}(u, v, q), q^{\lambda} ; s^{\lambda}, \mu, a\right) \geqq-N_{U}(\lambda)} \\
& \left.\quad \text { (= holding only if } N_{U}(\lambda)=0\right) .
\end{aligned}
$$

If $\bar{\gamma}(U)$ constant and $\bar{\lambda}(U)$ variable, every denominator factor of $\left(1-\hat{\tau}_{\lambda}\right) \hat{Y}_{\lambda}$ is independent of $q^{\lambda}$ and $\left(s^{\lambda}-1\right)$ or dependent on $v$, and $q^{\lambda}\left(k^{\gamma}(u, v, q), q^{\gamma}\right)$ is independent of $v$. Hence

$$
\begin{aligned}
& \frac{\operatorname{degr}}{\hat{u} \mid q^{\gamma} v\left(s^{\gamma}-1\right)} S_{\gamma}\left(1-\hat{\tau}_{\lambda}\right) \hat{Y}_{\lambda}\left(k^{\lambda}(u, v, q), q^{\lambda} ; s^{\lambda}, \mu, a\right) \\
& \quad \geqq \operatorname{degr}_{\hat{u} \mid q^{\lambda} v\left(s^{\lambda}-1\right)}\left(1-\hat{\tau}_{\lambda}\right) \hat{Y}_{\lambda}\left(k^{\lambda}(u, v, q), q^{\lambda} ; s^{\lambda}, \mu, a\right) \geqq-N_{U}(\lambda) \\
& \quad\left(=\text { holding only if } N_{U}(\lambda)=0\right) .
\end{aligned}
$$


Next, we prove the statements 2.c and 2.d of the lemma. Let $\bar{\gamma}(U)$ be constant. If $\bar{\lambda}(U)$ is constant, we get

$$
\underline{\operatorname{degr}}_{\widehat{u q}^{\lambda}\left(s^{\lambda}-1\right) \mid v} \hat{\tau}_{\lambda} \hat{Y}_{\lambda}\left(k^{\lambda}(u, v, q), q^{\lambda} ; s^{\lambda}, \mu, a\right) \geqq \max (0, \varrho(\lambda))-N_{U}(\lambda),
$$

where we have used Lemma 6.3.2. If $\bar{\lambda}(U)$ is variable, using Lemma 6.3.3, we have

$$
\underline{\operatorname{degr}} \widehat{u q}_{q^{\lambda}\left(s^{\lambda}-1\right) \mid v}\left(1-\hat{\tau}_{\lambda}\right) \hat{Y}_{\lambda}\left(k^{\lambda}(u, v, q), q^{\lambda} ; s^{\lambda}, \mu, a\right) \geqq \max (0, \varrho(\lambda))-N_{U}(\lambda) .
$$

Using the above mentioned property of the substitution operator $S_{\gamma}$, we get

$$
\begin{aligned}
& \operatorname{degr}_{\widehat{u q^{\gamma}\left(s^{\gamma}-1\right) \mid v}} S_{\gamma} \hat{\tau}_{\lambda} \hat{Y}_{\lambda}\left(k^{\lambda}(u, v, q), q^{\lambda} ; s^{\lambda}, \mu, a\right) \\
& \quad \geqq \underset{\operatorname{degr}}{\widehat{u q}^{\lambda}\left(s^{\lambda}-1\right) \mid v} \hat{\tau}_{\lambda} \hat{Y}_{\lambda}\left(k^{\lambda}(u, v, q), q^{\lambda} ; s^{\lambda}, \mu, a\right) \\
& \quad \geqq \max (0, \varrho(\lambda))-N_{U}(\lambda)
\end{aligned}
$$

and

$$
\begin{aligned}
& \operatorname{degr}_{\widehat{u q}^{\gamma}\left(s^{\gamma}-1\right) \mid v} S_{\gamma}\left(1-\hat{\tau}_{\lambda}\right) \hat{Y}_{\lambda}\left(k^{\lambda}(u, v, q), q^{\lambda} ; s^{\lambda}, \mu, a\right) \\
& \quad \geqq \underset{\operatorname{degr}}{\widehat{u q}^{\lambda}\left(s^{\lambda}-1\right) \mid v}\left(1-\hat{\tau}_{\lambda}\right) \hat{Y}_{\lambda}\left(k^{\lambda}(u, v, q), q^{\lambda} ; s^{\lambda}, \mu, a\right) \\
& \quad \geqq \max (0, \varrho(\lambda))-N_{U}(\lambda) .
\end{aligned}
$$

3. Let $\gamma \in U$ and $\gamma_{1}, \ldots, \gamma_{c}$ be the maximal elements of $U(\gamma)$. By hypothesis of induction, Lemma 6.4.2 holds, where $\lambda$ represents $\gamma_{1}, \ldots, \gamma_{c}$. We must show that $\gamma$ satisfies Lemma 6.4.1, which concludes the proof.

In general,

$$
\begin{aligned}
& \frac{\operatorname{degr}_{\hat{u} \mid q^{\gamma} v\left(s^{\gamma}-1\right)} \hat{Y}_{\gamma}\left(k^{\gamma}(u, v, q), q^{\gamma} ; s^{\gamma}, \mu, a\right)}{\geqq} \\
& \quad \frac{\operatorname{degr}_{\hat{u} \mid q q^{\gamma} v\left(s^{\gamma}-1\right)} \hat{I}_{\bar{\gamma}(U)}\left(k^{\gamma}(u, v, q), q^{\gamma} ; s^{\gamma}, \mu, a\right)}{\quad} \quad+\sum_{i=1}^{c} \operatorname{degr}_{\hat{u} \mid q^{\gamma} v\left(s^{\gamma}-1\right)} S_{\gamma} \hat{f}\left(\gamma_{i}\right) \hat{Y}_{\gamma_{t}}\left(k^{\gamma_{1}}(u, v, q), q^{\gamma_{2}} ; s^{\gamma_{l}}, \mu, a\right) \\
& \geqq 0+\sum_{i=1}^{c}\left(-N_{U}\left(\gamma_{i}\right)\right) \\
& \geqq-N_{U}(\gamma) \quad\left(=\text { holding only if } N_{U}(\gamma)=0\right),
\end{aligned}
$$
where we have used that $N_{U}(\gamma) \geqq \sum_{i=1}^{c} N_{U}\left(\gamma_{i}\right)$, and that all propagators of $\hat{I}_{\bar{\gamma}(U)}$
depend on $\left(s^{\gamma}-1\right)$.

Now let $\bar{\gamma}(U)$ be constant.

Let $\bar{\Sigma}_{1}, \ldots, \bar{\Sigma}_{b}$ be the mutually disjoint, connected and consequently 1PI subdiagrams of $\bar{\gamma}(U)$ spanned by the lines $L \in \mathscr{L}_{\bar{\gamma}(U)}$ satisfying $k_{L}^{\gamma}(0,0, q) \neq 0$. Using $\varrho(B) \leqq 4$ and $\varrho(B) \leqq r(B)$ for all vertices $B \in \mathscr{B}_{\gamma}$, and writing $T=\left(\gamma / \gamma_{1} \ldots \gamma_{c}\right) / \bar{\Sigma}_{1} \ldots \bar{\Sigma}_{b}$, we get

$$
\begin{aligned}
& \underline{\operatorname{degr}}_{\widehat{u q^{\gamma}\left(s^{\gamma}-1\right) \mid v}} \hat{Y}_{\gamma}\left(k^{\gamma}(u, v, q), q^{\gamma} ; s^{\gamma}, \mu, a\right) \\
& \geqq \underline{\operatorname{degr}}_{\widehat{u q^{\gamma}\left(s^{\gamma}-1\right) \mid v}} \hat{I}_{\bar{\gamma}(U)}\left(k^{\gamma}(u, v, q), q^{\gamma} ; s^{\gamma}, \mu, a\right) \\
& +\sum_{i=1}^{c} \underline{\operatorname{degr}}_{\widehat{u} q^{\gamma}\left(s^{\gamma}-1\right) \mid v} S_{\gamma} \hat{f}\left(\gamma_{i}\right) \hat{Y}_{\gamma_{l}}\left(k^{\gamma_{i}}(u, v, q), q^{\gamma_{z}} ; s^{\gamma_{2}}, \mu, a\right) \\
& \geqq \underline{\operatorname{degr}}_{\widehat{u q}^{\gamma}\left(s^{\gamma}-1\right)} \hat{I}_{T}\left(k^{\gamma}(u, 0,0), q^{\gamma} ; s^{\gamma}, \mu, a\right) \\
& +\sum_{i=1}^{c} \underline{\operatorname{degr}_{\widehat{u q} \widehat{q}^{\prime}\left(s^{\gamma}-1\right) \mid v}} S_{\gamma} \hat{f}\left(\gamma_{i}\right) \hat{Y}_{\gamma_{2}}\left(k^{\gamma_{2}}(u, v, q), q^{\gamma_{2}} ; s^{\gamma_{2}}, \mu, a\right)
\end{aligned}
$$




$$
\begin{aligned}
& \geqq r(T)-4 m(T)+\sum_{i=1}^{c} \max \left(0, \varrho\left(\gamma_{i}\right)\right)-\sum_{i=1}^{c} N_{U}\left(\gamma_{i}\right) \\
& \geqq \varrho(\gamma)-\left[4 m(\bar{\gamma}(U))+\sum_{i=1}^{c} N_{U}\left(\gamma_{i}\right)\right], \quad(\text { by Lemma 6.2) } \\
& =\varrho(\gamma)-N_{U}(\gamma) . \quad \square
\end{aligned}
$$

Finally, using Lemma 6.4 we can prove that the renormalized Feynman integrand $\hat{R}_{\Gamma}$ satisfies the IR-conditions (II).

Theorem 3. Let $\Gamma$ be a 1PI Feynman diagram having $m$ loops and

$$
u_{1}, \ldots, u_{r}, \quad v_{1}, \ldots, v_{d}
$$

$r+d=m$, be an arbitrary basis of $\mathscr{L}$, the set of all $l_{L}, L \in \mathscr{L}_{\Gamma}$ and of all $k_{L}^{\gamma}$ for all $1 \mathrm{PI}$ subdiagrams $\gamma$ of $\Gamma$ and $L \in \mathscr{L}_{\gamma}$. Let $H \in \mathscr{H}$ be given by variable $\left(v_{1}, \ldots, v_{d}\right)$ and constant $\left(u_{1}, \ldots, u_{r}\right)$, and $U$ a $\Gamma$-forest which is complete on $H$. Then $\hat{X}_{U}$ of Lemma 5.1 satisfies

$$
\left.\underline{\operatorname{degr}}_{\hat{u} \mid v} \hat{X}_{U}(k(u, v, q), q ; s, \mu, a)\right|_{s=1}>-4 r,
$$

hence

$$
\left.\underline{\operatorname{degr}}_{\hat{u} \mid v} \hat{R}_{\Gamma}(k(u, v, q), q ; s, \mu, a)\right|_{s=1}+4 r>0 .
$$

This means that the IR-convergence conditions (II) are satisfied, and Theorem 1 is proved.

Proof of Theorem 3. We must show that

$$
\left.\underline{\operatorname{degr}}_{\hat{u} \mid v}\left(1-\hat{\tau}_{\Gamma}\right) \hat{Y}_{\Gamma}\left(k^{\Gamma}(u, v, q), q^{\Gamma} ; s^{\Gamma}, \mu, a\right)\right|_{q^{I}=q, s^{\Gamma=1}}>-4 r .
$$

At first, note that the denominator of $\hat{\tau}_{\Gamma} \hat{Y}_{\Gamma}$ is independent of $q^{\Gamma}$ and of $s^{\Gamma}-1$. Using Lemma 6.4.2, we get

$$
\begin{aligned}
& \frac{\left.\operatorname{degr}_{\hat{u} \mid v} \hat{\tau}_{\Gamma} \hat{Y}_{\Gamma}\left(k^{\Gamma}(u, v, q), q^{\Gamma} ; s^{\Gamma}, \mu, a\right)\right|_{q^{\Gamma}=q, s^{\Gamma}=1}}{\quad \underline{\operatorname{degr}} \hat{u} \mid q^{\Gamma} v\left(s^{\Gamma}-1\right)} \hat{\tau}_{\Gamma} \hat{Y}_{\Gamma}\left(k^{\Gamma}(u, v, q), q^{\Gamma} ; s^{\Gamma}, \mu, a\right)>-4 r .
\end{aligned}
$$

All what remains to prove is that

$$
\left.\underline{\operatorname{degr}}_{\hat{u} \mid v} \hat{Y}_{\Gamma}\left(k^{\Gamma}(u, v, q), q^{\Gamma} ; s^{\Gamma}, \mu, a\right)\right|_{q^{\Gamma}=q, s^{\Gamma}=1}>-4 r .
$$

Let $\gamma_{1}, \ldots, \gamma_{c}$ be the maximal elements of $U(\Gamma)$, so that

$$
\hat{Y}_{\Gamma}\left(k^{\Gamma}, q^{\Gamma} ; s^{\Gamma}, \mu, a\right)=\hat{I}_{\bar{\Gamma}(U)}\left(k^{\Gamma}, q^{\Gamma} ; s^{\Gamma}, \mu, a\right)+\sum_{i=1}^{c} S_{\Gamma} \hat{\tau}_{\gamma_{t}} \hat{Y}_{\gamma_{i}}\left(k^{\gamma_{2}}, q^{\gamma_{2}} ; s^{\gamma_{2}}, \mu, a\right)
$$

1. Suppose that $\bar{\Gamma}(U)$ is variable. Then $N_{U}(\Gamma)=\sum_{i=1}^{c} N_{U}\left(\gamma_{i}\right) \leqq 4 r(=$ holding only if $N_{U}(\Gamma) \neq 0$ ). Using

$$
\underline{\operatorname{degr}}_{\hat{u} \mid v} \hat{I}_{\bar{\Gamma}(U)}\left(k^{\Gamma}(u, v, q), q^{\Gamma} ; s^{\Gamma}, \mu, a\right) \geqq 0
$$


and Lemma 6.4.2, we get

$$
\begin{aligned}
& \left.\operatorname{degr}_{\hat{u} \mid v} \hat{Y}_{\Gamma}\left(k^{\Gamma}(u, v, q), q^{\Gamma} ; s^{\Gamma}, \mu, a\right)\right|_{q^{\Gamma}=q, s^{\Gamma}=1} \\
& \geqq\left.\operatorname{degr}_{\hat{u} \mid v} \hat{I}_{\bar{\Gamma}(U)}\left(k^{\Gamma}(u, v, q), q^{\Gamma} ; s^{\Gamma}, \mu, a\right)\right|_{q^{\Gamma}=q, s^{\Gamma}=1} \\
& \quad+\sum_{i=1}^{c} \underline{\operatorname{degr}_{\hat{u} \mid q^{\Gamma} v\left(s^{\Gamma}-1\right)} S_{\Gamma} \hat{\tau}_{\gamma_{i}} \hat{Y}_{\gamma_{i}}\left(k^{\gamma_{2}}(u, v, q), q^{\gamma_{2}} ; s^{\gamma_{2}}, \mu, a\right)>-4 r .}
\end{aligned}
$$

2. Suppose that $\bar{\Gamma}(U)$ is constant.

Take all mutually disjoint, connected subdiagrams $\bar{\Lambda}_{0}, \ldots, \bar{\Lambda}_{b}$ of $\tilde{\Gamma} / \gamma_{1} \ldots \gamma_{c}$ spanned by the $q$-lines and all lines $L \in \mathscr{L}_{\vec{\Gamma}(U)}$ satisfying

$$
k_{L}(0,0, q)+q_{L}(q) \neq 0 .
$$

Furthermore, let $\lambda_{1}, \ldots, \lambda_{e}$ be the elements of $\left\{\gamma_{1}, \ldots, \gamma_{c}\right\}$ which corresponds in $\widetilde{\Gamma} / \gamma_{1}, \ldots, \gamma_{c}$ to vertices not contained in $\mathscr{B}_{\bar{A}_{0}} \cup \ldots \cup \mathscr{B}_{\bar{A}_{b}}$, and let $\varrho_{1}, \ldots, \varrho_{l} \in\left\{\gamma_{1}, \ldots, \gamma_{c}\right\}$ the remaining ones, i.e.

$$
\begin{aligned}
& \left\{\varrho_{1}, \ldots, \varrho_{l}\right\} \cup\left\{\lambda_{1}, \ldots, \lambda_{e}\right\}=\left\{\gamma_{1}, \ldots, \gamma_{c}\right\} \quad l+e=c . \\
& \left\{\varrho_{1}, \ldots, \varrho_{l}\right\} \cap\left\{\lambda_{1}, \ldots, \lambda_{e}\right\}=\emptyset,
\end{aligned}
$$

At first, the following lower bounds on the IR-degrees can be given.

a) The denominator of $S_{\Gamma} \hat{\tau}_{\underline{\varrho}_{\imath}} \hat{Y}_{\varrho_{\imath}}$ is independent of $q^{\Gamma}$ and $s^{\Gamma}-1$, hence

$$
\begin{aligned}
& \left.\operatorname{degr}_{\hat{u} \mid v} S_{\Gamma} \hat{\tau}_{\varrho_{\imath}} \hat{Y}_{\varrho_{i}}\left(k^{\varrho_{\imath}}(u, v, q), q^{Q_{\imath}} ; s^{\varrho_{\imath}}, \mu, a\right)\right|_{q^{\Gamma}=q, s^{I}=1}
\end{aligned}
$$

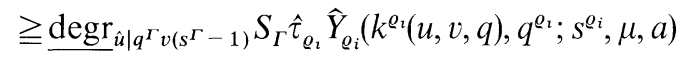

$$
\begin{aligned}
& \geqq-N_{U}\left(\varrho_{i}\right) \quad\left(=\text { holding only if } N_{U}\left(\varrho_{i}\right)=0\right) \text {, (by Lemma 6.4). }
\end{aligned}
$$

b) For $\bar{\varrho}_{i}$ variable, using the same arguments as for (6.17),

$$
\begin{aligned}
& \left.\underline{\operatorname{degr}}_{\hat{u} \mid v} S_{\Gamma}\left(1-\hat{\tau}_{\varrho_{\imath}}\right) \hat{Y}_{\varrho_{\imath}}\left(k^{\varrho_{i}}(u, v, q), q^{\varrho_{2}} ; s^{\varrho_{u}}, \mu, a\right)\right|_{q \Gamma=q, s^{\Gamma}=1} \\
& \geqq \underline{\operatorname{degr}}_{\hat{u} \mid q^{\Gamma} v\left(s^{\Gamma-1)}\right.} S_{\Gamma}\left(1-\hat{\tau}_{\varrho_{\imath}}\right) \hat{Y}_{\varrho_{\imath}}\left(k^{\varrho_{\imath}}(u, v, q), q^{\varrho_{\imath}} ; s^{\varrho_{\imath}}, \mu, a\right) \\
& \geqq-N_{U}\left(\varrho_{i}\right) \quad\left(=\text { holding only if } N_{U}\left(\varrho_{i}\right)=0\right), \quad \text { (by Lemma 6.4). }
\end{aligned}
$$

c) For every $\lambda_{1}, \ldots, \lambda_{e}$

$$
q^{\lambda_{2}}=\left.q^{\lambda_{2}}\left(k^{\Gamma}(u, v, q), q^{\Gamma}\right)\right|_{q^{\Gamma}=q}=q^{\lambda_{2}}(u)
$$

is a linear function, hence

$$
\begin{aligned}
& \left.\operatorname{degr}_{\hat{u} \mid v} S_{\Gamma} \hat{\tau}_{\lambda_{\imath}} \hat{Y}_{\lambda_{\imath}}\left(k^{\lambda_{2}}(u, v, q), q^{\lambda_{\imath}} ; s^{\lambda_{i}}, \mu, a\right)\right|_{q^{\Gamma}=q, s^{\Gamma-1}=0} \\
& \geqq \underline{\operatorname{degr}}{\widehat{u q q^{\lambda_{1}}\left(s^{\lambda_{i}}-1\right) \mid v}} \hat{\tau}_{\lambda_{l}} \hat{Y}_{\lambda_{i}}\left(k^{\lambda_{i}}(u, v, q), q^{\lambda_{i}} ; s^{\lambda_{2}}, \mu, a\right) \\
& \geqq \max \left(0, \varrho\left(\lambda_{i}\right)\right)-N_{U}\left(\lambda_{i}\right), \quad \text { (by Lemma 6.4) }
\end{aligned}
$$

and for $\bar{\lambda}_{i}(U)$ variable

$$
\begin{aligned}
& \left.\underline{\operatorname{degr}}_{\hat{u} \mid v} S_{\Gamma}\left(1-\hat{\tau}_{\lambda_{i}}\right) \hat{Y}_{\lambda_{i}}\left(k^{\lambda_{2}}(u, v, q), q^{\lambda_{2}} ; s^{\lambda_{2}}, \mu, a\right)\right|_{q^{\Gamma}=q, s^{\Gamma-1}=0} \\
& \geqq \underline{\operatorname{degr}}_{u q^{\lambda_{1}}\left(s^{\lambda i}-1\right) \mid v}\left(1-\hat{\tau}_{\lambda_{\imath}}\right) \hat{Y}_{\lambda_{\imath}}\left(k^{\lambda_{i}}(u, v, q), q^{\lambda_{2}} ; s^{\lambda_{2}}, \mu, a\right) \\
& \geqq \max \left(0, \varrho\left(\lambda_{i}\right)\right)-N_{U}\left(\lambda_{i}\right) \quad \text { (by Lemma 6.4). }
\end{aligned}
$$


Next, we use the inequalities a)-c) to conclude the proof of Theorem 3. By assumption, the external momenta of $\Gamma$ are non-exceptional, and for every internal vertex $B \in \mathscr{B}_{\Gamma}$, we have $r(B) \geqq \varrho(B)=4$. Applying Lemma 6.1 to $T=\left(\tilde{\Gamma} / \gamma_{1} \ldots \gamma_{c}\right) / \bar{\Lambda}_{0} \ldots \bar{\Lambda}_{b}$, we get

$$
\begin{aligned}
& \left.\operatorname{degr}_{\hat{u} \mid v} \hat{Y}_{\Gamma}\left(k^{\Gamma}(u, v, q), q^{\Gamma} ; s^{\Gamma}, \mu, a\right)\right|_{q^{\Gamma}=q, s^{\Gamma}=1} \\
& \geqq\left.\underline{\operatorname{degr}_{\hat{u}} \mid v \hat{I}_{\bar{I}(U)}}\left(k^{\Gamma}(u, v, q), q ; s^{T}, \mu, a\right)\right|_{s^{\Gamma}=1} \\
& +\left.\sum_{i=1}^{l} \underline{\operatorname{degr}}_{\hat{u} \mid v} S_{\Gamma} \hat{f}\left(\varrho_{i}\right) \hat{Y}_{\varrho_{\imath}}\left(k^{\varrho_{i}}(u, v, q), q^{\varrho_{i}} ; s^{\varrho_{i}}, \mu, a\right)\right|_{q^{\gamma}=q, s^{\Gamma}=1} \\
& +\left.\sum_{i=1}^{e} \underline{\operatorname{degr}}_{\hat{u} \mid v} S_{\Gamma} \hat{f}\left(\lambda_{i}\right) \hat{Y}_{\lambda_{\imath}}\left(k^{\lambda_{i}}(u, v, q), q^{\lambda_{i}} ; s^{\lambda_{2}}, \mu, a\right)\right|_{q^{\Gamma}=q, s^{\Gamma}=1} \\
& \geqq \underline{\operatorname{degr}_{\hat{u}}} \hat{I}_{T}\left(k^{\Gamma}(u, v=0, q=0), q=0 ; s=1, \mu, a\right) \\
& +\sum_{i=1}^{l}\left(-N_{U}\left(\varrho_{i}\right)\right)+\sum_{i=1}^{e}\left[\max \left(0, \varrho\left(\lambda_{i}\right)\right)-N_{U}\left(\lambda_{i}\right)\right] \\
& =r(T)-4 m(T)+\sum_{i=1}^{e} \max \left(0, \varrho\left(\lambda_{i}\right)\right)-\sum_{i=1}^{c} N_{U}\left(\gamma_{i}\right) \\
& >-\left[4 m(\bar{\Gamma}(U))+\sum_{i=1}^{c} N_{U}\left(\gamma_{i}\right)\right] \text { (by Lemma 6.1) } \\
& =-N_{U}(\Gamma) \geqq-4 r \text {. }
\end{aligned}
$$

\section{Conclusions}

We have proposed a renormalization procedure for lattice Feynman integrals which applies also in presence of zero-mass propagators. The method is a fusion of the lattice version of the BPHZ renormalization prescription [4] and the auxiliary mass method of Lowenstein and Zimmermann $[9,10]$. It applies to a wide class of lattice field theories. Under very general conditions, the renormalized theory is IRconvergent for every finite lattice spacing, and the continuum limit exists. The set of renormalizable, IR-finite theories is constrained by the condition that all vertices should have an IR-degree not less than four. Apart from the possibility of massless propagators, the assumptions on the structure of momentum space Feynman integrals are the same as in the massive case [4]. The integrand should be periodic with the Brillouin zone in all the momenta, a property which is reflected by the fact that the counterterms are also periodic. The propagators are assumed to have only one pole in the Brillouin zone. In particular, the renormalization program does not work for lattice fermions whose propagators have poles on the boundary of the Brillouin zone. Furthermore, the integrand should be differentiable to such a degree that all subtractions necessary to subtract the divergencies can be done without problems.

With respect to universality of perturbation theory and power counting renormalizability, the same arguments as in the massive case [4] apply also to massless lattice field theories. The continuum limit of the renormalized theory does not depend on the specific choice of the lattice action. It is given by the continuum 
field theory which is described by the (naive) continuum limit of the lattice action and is renormalized by the BPHZL finite part prescription $[9,10]$. Furthermore, if all coupling constants are dimensionless, a lattice field theory is renormalizable by power counting if and only if its (formal) continuum limit is renormalizable. Also, the counterterm philosophy is the same as in the massive case. After some symmetrizations with respect to the external momenta of the Green functions (as described in [4, Sect. 4]), the subtractions can always be written as counterterm contributions to the lattice action, which can be chosen to be local.

Actually, we have defined the renormalization procedure for scalar fields only. This we have done to simplify the notation. There is no problem to generalize the method to fields carrying internal symmetries and spin. This can be done by introducing so-called index-distributions in Feynman integrals [4] which associate every line ending with a set of symmetry labels. The definitions (2.27), (2.28) of divergence degrees are replaced by

$$
\begin{aligned}
& \omega\left(\hat{\Delta}_{L}\right)=\max _{\alpha_{L}, \beta_{L}} \overline{\operatorname{degr}}_{\hat{l}_{L} s} \hat{\Delta}_{L}\left(l_{L}, \alpha_{L}, \beta_{L} ; s, \mu, a\right), \\
& r\left(\hat{\Delta}_{L}\right)=\min _{\alpha_{L}, \beta_{L}} \underline{\operatorname{degr_{r_{L}}(s-1)}} \hat{\Delta}_{L}\left(l_{L}, \alpha_{L}, \beta_{L} ; s, \mu, a\right)
\end{aligned}
$$

for every line $L$ and

$$
\begin{aligned}
\omega\left(\hat{V}_{B}\right) & =\max _{\left\{\alpha_{L}\right\}_{B}} \overline{\operatorname{degr}}_{\widehat{l}_{\left.L_{L}\right\}_{B}}{ }} \hat{V}_{B}\left(\left\{l_{L}, \alpha_{L}\right\}_{B} ; s, \mu, a\right), \\
r\left(\hat{V}_{B}\right) & =\min _{\left\{\alpha_{L}\right\}_{B}} \operatorname{degr}_{\widehat{l}_{\left.l_{L}\right\}_{B}(s-1)}} \hat{V}_{B}\left(\left\{l_{L}, \alpha_{L}\right\}_{B} ; s, \mu, a\right)
\end{aligned}
$$

for every vertex $B$, where the maxima and minima are over all possible symmetry labels of line endings at the vertex $B$.

Renormalized Green functions depend on the auxiliary masses introduced by the subtraction scheme. This dependence may be absorbed by additional finite renormalizations satisfying the IR-constraints and leading to equivalent renormalization schemes. For instance, by an appropriate choice, this corresponds to subtractions at non-exceptional momenta plus additional finite renormalizations at vanishing momentum for two- and three-point functions necessary to get IR-finite amplitudes in higher orders. In most applications, these additional subtractions are needed only for diagrams with two or three massless external lines.

The renormalization program proposed here applies also to lattice gauge field theories. After convenient gauge fixing, such a theory is perturbatively renormalizable by power counting, i.e. with increasing number of loops the order of subtractions needed does not increase, and the continuum limit of the renormalized theory exists. The counterterms needed can always be chosen to be local. A priori, only little can be said about their structure. However, if there exists a BRS-symmetry on the lattice, the Green functions satisfy the corresponding Slavnov-identities. It then should be possible to show that to every order, the counterterms needed are of a restricted form which allows the theory to be renormalized simply by renormalizing the parameters in the original lattice action. 


\section{Appendix. Properties of Lattice Degrees}

In this appendix we list those properties of the IR- and UV-degrees defined in Sect. 2.3 which are permanently used in the text without explicit reference. They are direct consequences of the definitions.

Let $V \in \mathscr{C}^{c}$ be of the form (2.18). Then

$$
\begin{aligned}
& \left.\underline{\operatorname{degr}}_{\widehat{u} q(s-1) \mid v} V(u, v, q, \bar{q} ; s, \mu, a)\right|_{q=0, s-1=0} \geqq \underline{\operatorname{degr}}_{\widehat{u} q(s-1) \mid v} V(u, v, q, \bar{q} ; s, \mu, a), \\
& \left.\underline{\operatorname{degr}}_{\hat{u} \mid q v(s-1)} V(u, v, q, \bar{q} ; s, \mu, a)\right|_{q=0, s-1=0} \underline{\operatorname{degr}}_{\hat{u} \mid q v(s-1)} V(u, v, q, \bar{q} ; s, \mu, a),
\end{aligned}
$$

and

$$
\begin{aligned}
&\left.\overline{\operatorname{degr}}_{\hat{v}} V(u, v, q, \bar{q} ; s, \mu, a)\right|_{q=0, s=0}=\left.\overline{\operatorname{degr}}_{\hat{v} \underline{ }} V(u, v, q, \bar{q} ; s, \mu, a)\right|_{q=0, s=0} \\
& \leqq \overline{\operatorname{degr}}_{\hat{v} \hat{q} s} V(u, v, q, \bar{q} ; s, \mu, a), \\
&\left.\overline{\operatorname{degr}}_{\hat{v}} V(u, v, q, \bar{q} ; s, \mu, a)\right|_{q=0, s=0} \leqq \overline{\operatorname{degr}}_{\hat{v}} V(u, v, q, \bar{q} ; s, \mu, a) .
\end{aligned}
$$

The degrees of derivatives and of sums and products of functions of the function class $\mathscr{F}$ satisfy inequalities which are direct generalizations of the corresponding inequalities for the UV- and IR-degrees with respect to the momenta only, as given in [3, Lemma 2.2] and [6, Lemma 2.1], respectively.

Let $F, F_{1}, \ldots, F_{e}$ be of the form (2.19). Then

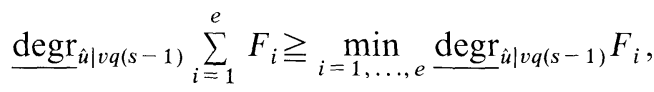

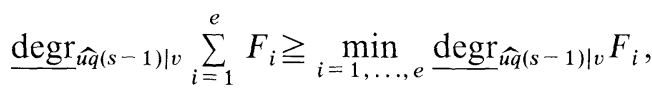

$$
\begin{aligned}
& \underline{\operatorname{degr}}_{\hat{u} \mid v q(s-1)} \prod_{i=1}^{e} F_{i} \geqq \sum_{i=1}^{e} \underline{\operatorname{degr}_{\hat{u} \mid v q(s-1)}} F_{i}, \\
& \underline{\operatorname{degr}}_{\widehat{u} \hat{q}(s-1) \mid v} \prod_{i=1}^{e} F_{i} \geqq \sum_{i=1}^{e} \underline{\operatorname{degr}}_{\widehat{u} \hat{q}(s-1) \mid v} F_{i},
\end{aligned}
$$

and

$$
\begin{aligned}
& \overline{\operatorname{degr}}_{\widehat{v q} s} \sum_{i=1}^{e} F_{i} \leqq \max _{i=1, \ldots, e} \overline{\operatorname{degr}}_{\widehat{v} \hat{q} s} F_{i}, \\
& \overline{\operatorname{degr}}_{\widehat{v q} s} \prod_{i=1}^{e} F_{i} \leqq \sum_{i=1}^{e} \overline{\operatorname{degr}}_{\widehat{v q} s} F_{i} .
\end{aligned}
$$

Furthermore, for every $b \in \mathbb{N}_{0}=\{0,1,2, \ldots\}$ and $l=\left(l_{1}, \ldots, l_{w}\right) \in \mathbb{N}_{0}^{w}$, we have

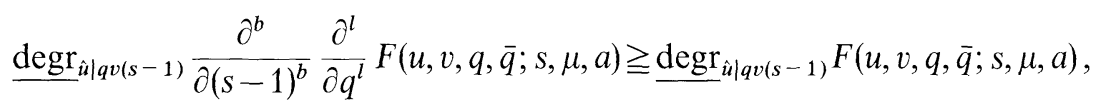
$\underline{\operatorname{degr}}_{\hat{u} \hat{q}(s-1) \mid v} \frac{\partial^{b}}{\partial(s-1)^{b}} \frac{\partial^{l}}{\partial q^{l}} F(u, v, q, \bar{q} ; s, \mu, a) \geqq \underline{\operatorname{degr}}_{\widehat{u} q(s-1) \mid v} F(u, v, q, \bar{q} ; s, \mu, a)-(b+|l|)$, and

$$
\begin{aligned}
\overline{\operatorname{degr}}_{\hat{v}} \frac{\partial^{b}}{\partial s^{b}} \frac{\partial^{l}}{\partial q^{l}} F(u, v, q, \bar{q} ; s, \mu, a) \leqq \overline{\operatorname{degr}}_{\hat{v}} F(u, v, q, \bar{q} ; s, \mu, a), \\
\overline{\operatorname{degr}}_{\widehat{v} \hat{q} s} \frac{\partial^{b}}{\partial s^{b}} \frac{\partial^{l}}{\partial q^{l}} F(u, v, q, \bar{q} ; s, \mu, a) \leqq \overline{\operatorname{degr}}_{\widehat{v q} s} F(u, v, q, \bar{q} ; s, \mu, a)-(b+|l|),
\end{aligned}
$$

where $|l|=\sum_{i=1}^{w} l_{i}$ 
Acknowledgement. I am grateful to Martin Lüscher for many discussions.

\section{References}

1. Weinberg, S.: High energy behaviour in quantum field theory. Phys. Rev. 118, 838-849 (1960)

2. Hahn, Y., Zimmermann, W.: An elementary proof of Dyson's power counting theorem. Commun. Math. Phys. 10, 330-342 (1968)

3. Reisz, T.: A power counting theorem for Feynman integrals on the lattice. Commun. Math. Phys. 116, 81-126 (1988)

4. Reisz, T.: Renormalization of Feynman integrals on the lattice. Commun. Math. Phys.,(1988) (in press)

5. Zimmermann, W.: Convergence of Bogoliubov's method of renormalization in momentum space. Commun. Math. Phys. 15, 208-234 (1969)

6. Reisz, T.: A convergence theorem for lattice Feynman integrals with massless propagators. Commun. Math. Phys. 116, 573-606 (1988)

7. Lowenstein, J.H., Zimmermann, W.: The power counting theorem for Feynman integrals with massless propagators. Commun. Math. Phys. 44, 73-86 (1975)

8. Bandelloni, G., Becchi, C., Blasi, A., Collina, R.: Renormalization of models with radiative mass generation. Commun. Math. Phys. 67, 147-178 (1978)

9. Lowenstein, J.H., Zimmermann, W.: On the formulation of theories with zero-mass propagators. Nucl. Phys. B 86, 77 (1975)

10. Lowenstein, J.H.: Convergence theorems for renormalized Feynman integrals with zero-mass propagators. Commun. Math. Phys. 47, 53-68 (1976)

11. Symanzik, K.: Small-distance behaviour in field theory. Springer Tracts in Modern Physics, Vol. 57, p. 222. Berlin, Heidelberg, New York: Springer 1971

Communicated by A. Jaffe

Received October 29, 1987

Note added in proof. The constraints on the IR-degrees of the vertices imposed by the convergence theorems of Sect. 3 may be relaxed somewhat [10]. Namely, there may be at most one internal vertex $B$ with an IR-degree $r(B)$ equal to 3 . Correspondingly, the IR-subtraction degree $\varrho(B)$ of that vertex must also be equal to 3, i.e.

$$
r(B)=\sigma(B)=3 .
$$

(If the external momenta are integrated over, then $3 \leqq \varrho(B) \leqq r(B)$ ). That is possible because the IR-dimensions of the fields satisfy $r_{k} \geqq 1$. All statements remain true with this modification, and the only change is in the proof of Lemma 6.1.

The generalization will be useful in applications. 
